\title{
Some non-standard interpretations of the axiomatic basis of Leśniereskis Ontology
}

\author{
RAFAe URBANIAK* \\ Department of Philosophy, University of Calgary \\ Calgary, Alberta t2n in4, Canada \\ rafal.urbaniak@ucalgary.ca \\ Received by Greg Restall \\ Published January 25, 2006 \\ http://www.philosophy.unimelb.edu.au/ajl/2006 \\ (c) 2006 Rafał Urbaniak
}

\begin{abstract}
We propose an intuitive understanding of the statement: 'an axiom (or: an axiomatic basis) determines the meaning of the only specific constant occurring in it.' We introduce some basic semantics for functors of the category $\frac{s}{n, n}$ of Leśniewski's Ontology. Using these results we prove that the popular claim that the axioms of Ontology determine the meaning of the primitive constants is false.
\end{abstract}

\section{PRELIMINARIES}

\section{I.I PURPOSE}

Intuitively, in a slogan, when we give axioms for a given axiomatic system one of our purposes is to characterize constants occurring in these axioms. Following this idea, axioms of Leśniewski's Ontology aim to characterize 'univocally' the primitive constants of this system. Usually, there is only one such a constant specific to Ontology; it is $\mathcal{\varepsilon}$ (sometimes, there are other constants: see Lejewski [9]). Hence, Lejewski writes:

In the original system of Ontology ... the meaning of the copula 'is'

(' $\varepsilon$ ' in symbols) is determined axiomatically ... [IO, p. 323]

Our purpose will be to investigate, whether in fact axiomatizations of Ontology determine a unique semantic interpretation of the primitive constant(s) of this

\footnotetext{
${ }^{*}$ I would like to thank Dr Jarosław Mrozek, Magda Baranowska, Adam Trybus-these are people who have inspired my work in the last year. I am grateful for remarks addressed to me by: Prof. A. W. Mostowski, Dr Richard Zach, and an anonymous referee of the Australasian fournal of Logic. Please address any remarks to: ninghijz@wp.pl
} 
axiomatizations. In order to proceed, we shall (i) introduce the language we will be talking about (ii) say what axioms and rules of inference were accepted in Ontology in some axiomatizations, (iii) present some possible interpretations of quantifiers in Ontology, (iv) explain what is meant by 'semantic interpretation of a given functor', and, when it will be done, (v) obtain the answer for the main problem.

\section{I.2 BASIC LANGUAGE (BL)}

In our deliberations we will use a basic language which is somewhat simpler than the full language of Ontology. It will nevertheless allow us to give a short statement of the essential results of our paper. We will suggest a possible way of extending our results to a richer language of Ontology. The presentation of our language is a simplified version of the language presented in another paper [2I].

Let $\left\{a, b, c, a_{1}, \ldots, a_{n}, b_{1}, \ldots, b_{n}, c_{1}, \ldots, c_{n}\right\}$ be a set of name variables of Ontology, say V. Next, we apply the following convention for variables in metalanguage: $\chi, \chi_{1}, \ldots, \chi_{n}$ represent sentential-expressions (including sentences and sentential formulae); $\mu, v, \mu_{1}, v_{1}, \ldots, \mu_{n}, v_{n}$ represent name-variables. $\int^{n}$ Let also $f, f_{1}, \ldots, f_{n}, g, g_{1}, \ldots, g_{n}$ represent functors of category $\frac{s}{n_{1} \ldots, n}$. Sometimes we use in the meta-language other variables-their usage should be selfexplanatory.

We introduce the following language. Our language $\mathrm{L}^{f}$ is relativized to a primitive functor $f$.

DEFINITION I $L^{f}=\left\langle\mathrm{FS}^{f}, \mathrm{~V}, \mathrm{f}, \wedge, \neg, \forall\right\rangle$

where $V$ is the already mentioned set of name variables of $L^{f}, f$ is a primitive functor of category $\frac{s}{n_{1}, n_{2}} ; \neg, \wedge$ are classical extensional functors of Sentential Calculus, here treated as primitive, $\forall$ is simply the universal quantifier. We omit the definitions of $\exists, \vee, \rightarrow$, $\equiv$ by means of $\wedge, \neg, \forall$, as any appropriate definitions suffice.

$F S^{f}$ is the union of $S^{f}$, the set of sentences $2^{2}$ of $L^{f}$, together with $F^{f}$, the set of propositional formulae of $L^{f}$. This means means that $\mathrm{FS}^{f}$ is the least set satisfying the following conditions:

I. If $\mu_{1}, \mu_{2} \in \mathrm{V}$ then $\left\ulcorner\mu_{1} f \mu_{2}\right\urcorner \in \mathrm{FS}^{f}$,

2. If $\left\ulcorner\mathrm{X}_{1}\right\urcorner,\left\ulcorner\mathrm{X}_{2}\right\urcorner \in \mathrm{FS}^{f}$ then $\left\ulcorner\neg \mathrm{X}_{1}\right\urcorner,\left\ulcorner\mathrm{X}_{1} \wedge \mathrm{X}_{2}\right\urcorner \in \mathrm{FS}^{f}$,

3. If $\ulcorner\mu\urcorner \in \mathrm{V}$ and $\ulcorner\chi\urcorner \in \mathrm{FS}^{f}$ then $\left\ulcorner\forall_{\mu} \chi\right\urcorner \in \mathrm{FS}^{f}$.

${ }^{\mathrm{I}}$ We also use the symbols of the same shape as logical constants in meta-language for naming these constants.

${ }^{2}$ For convenience, we do not distinguish between sentences and propositions. 
For the sake of simplicity BL does not contain variables of any category other than $n$, or constants other than name constants (introduced by means of the rule of definition) or functors of categories: $\frac{s}{n, n}, \frac{s}{s}, \frac{s}{s, s}$.

\section{I.3 INTERPRETATION OF QUANTIFIERS}

The problem of interpretation of quantifiers in Ontology was discussed in the technical literature [6, 77, I, [2]. There is no unique solution upon which logicians would agree (though much of the disagreement concerns technicalities).

The reason for such a controversy is the fact that the particular quantifier cannot be interpreted existentially (the meaning of the universal quantifier is also debatable, because of this) since we can prove in Ontology:

$$
\exists_{\mathrm{a}} \neg \operatorname{ex}(\mathrm{a})
$$

where 'ex' is to be read: 'exists' [7, p. 315]. Therefore different ideas of interpreting quantifiers have been suggested. We list some of them below:3

I. Meta-language interpretations: Ruth Barcan Marcus [I2, p. 252253] suggests that ' $\exists x \mathrm{Fx}$ ' is to be read 'Some substitution instance of ' $\mathrm{Fx}$ ' is true'. Following this idea, Küng distinguishes between substitutional and Leśniewskian reading of quantifiers:

(a) Substitutional REAding: $\exists_{a} \phi(a)$ is to be read:

If some $\alpha$ of the same category as $a$ is taken to be substituted for the inscription equiform to $a$, the following is asserted: $\phi(\alpha)$.

(b) LEŚNIEWSKIAN READING: $\exists_{a} \phi(a)$ is to be read:

For some extension which the inscriptions equiform with $a$ is taken to have, the following is asserted: $\phi(\alpha)$.

2. REFERENTIAL/OBJECTUAL INTERPRETATIONS: Kielkopf distinguishes some other interpretations of quantifiers which do not involve metalanguage [6].

(a) REFERENTIAL/OBJECTUAL INTERPRETATION: It consists in giving a set of objects, and claiming that quantifiers refer to them, in the sense, that $\exists_{a} \phi(a)$ is to be read:

For some object $\alpha$ of the given domain, $\phi(\alpha)$

${ }^{3}$ While discussing the interpretation of quantifiers I could not use two important papers on this issue $[\mathrm{I5}$, I6]. I regret that these papers were unavailable to me in the time of writing this paper. 
(b) REFERENTIAL/NON-OBJECTUAL INTERPRETATION: We choose a domain $D$ and associate with $D$ a non-empty set $R$ called the realm. The elements of $R$ are not viewed as existing objects. They are viewed as mind-dependent entities and not 'fully existing objects'. Then, a language is given an referential-non objectual interpretation iff it is given a quasi-referential interpretation, as if $R$ was a domain, but the set of existing objects is D.

3. MODEL-THEORETIC inteRPRETATION: Urbaniak [2I]. We indicate a domain of $\mathrm{D}$, considered to be the set of existing objects. The function of valuation on name-variables takes subsets of $D$ as values. If a name is considered empty, then its denotation is $\emptyset$, the empty set.

Let us elaborate a while on the given possibilities of interpreting quantifiers. We shall not consider how far a given interpretation is Leśniewskian. Instead, we shall give some arguments that for our purpose it suffices to accept the model-theoretic interpretation.

First, let us consider the substitutional interpretation. It is not quite clear which names may be substituted for name variables. There are no 'simple names'. Names always belong to some language. Therefore, such an interpretation of quantifiers either forces us to relativize our understanding of quantifiers to a given language, or requires that our quantifying involves implicit quantifying over languages (for example, we could read a universal quantification over a name variable as 'for any name in any language ...'). Moreover, it is far from being obvious that it is a really general understanding of quantifiers. What do we mean? That we can be lacking names for extensions.

To any meaningful name we can attribute a set that is its extension. In this way, every tautology (or valid expression) in the model-theoretic sense is a tautology (valid formula) in the substitutional sense. The question is, whether the implication in the other direction is true. The answer would be simple, if we assumed the condition that for any possible extension there is a name. Practically, it seems, however, that we are lacking names. As Ajdukiewicz argues [I, p. 138]:

Names of each language divide into simple and composed. There is always a finite number of the simple ones, the composite names are always finite combinations of simple names, hence names are countably infinite in number.

If we simply take a universe containing the set of natural numbers (or any other universe of the power $\aleph_{0}$ ), according to Cantor's Theorem, the number of subsets of the universe will be greater than $\boldsymbol{x}_{0}$. Hence, we would not have enough names to name all subsets, though each such a subset is a good candidate to be an extension of a name. Nevertheless, we can claim the following. If language L fulfills the following requirement: 
For any formula of elementary theory of numbers, if this formula contains exactly one free variable, there is in $\mathrm{L}$ a general name, extension of which is identical with the set of numbers satisfying this formula. ${ }^{4}$

then it is true, that any formula of L valid in lexical sense, is valid in the semantic sense.

The proof (which will not be described here fully) is based on the following result of Hilbert and Bernays:

Each formula of predicate calculus with identity, which is satisfied in some model, is also satisfied in a model in which the universe is the set of natural numbers. Relations (including sets treated as unary relations) attributed to predicates by interpretation are designed by open formulae of elementary theory of numbers.

More remarks on this problem and the full proof of the above theorem can be found in Pietruszczak [I3].

From what has been said, it follows that if we accept the model-theoretic interpretation, there is no loss of accuracy (in comparison with the substitutional interpretation).

A similar situation occurs in the case of so-called Leśniewskian interpretation. Since each possible extension of a meaningful name is a set, the modeltheoretical interpretation does a good job. Moreover, it is not quite clear what the essential difference between this interpretation and the model-theoretic one-in terms of truth conditions_-amounts to 5 Küng himself seems to agree that his interpretation implicitly contains the model-theoretical one:

... general procedure for reading all the functors of Leśniewski's 'ontology' in a way which is specifically Leśniewskian, ... which has at the same time the merit that it 'implicitly' contains the settheoretical interpretation of those functors. [7, p. 315]

Nevertheless, Küng's interpretation may have some virtues that the modeltheoretical interpretation lacks:

In my opinion the question of how to read quantified statements is of some consequence. The habit of giving merely model-theoretic interpretations and no intuitive paraphrases has tended to obscure some subtle, but very important aspects of oblique speech. [7, p. 309]

${ }^{4}$ Elementary theory of numbers is what we can say about natural numbers in terms of addition, multiplication, identity, sentence connectors, quantifiers quantifying over natural numbers, and the names of natural numbers, without introducing the notion of set.

${ }^{5}$ To some degree it may depend on whether we accept that there can be extensions of no actual names. This decision makes Leśniewskian interpretation either equivalent to the substitutional one, or to the model-theoretic one. 
In this paper we are not interested in aspects of oblique speech and we need a simple and user-friendly semantics. Fortunately, model theory formulated in the frame of standard set theory comes to the rescue ${ }^{6}$

Referential-objectual interpretation does not work, as far as we would like the valuation function to be from name-variables into the domain of object considered as existing individuals. First, it would lead to existential interpretation of particular quantifier. Next - it is not sure, how to interpret name-variables as semantically connected with exactly one existing object.

Nevertheless, the main idea of this interpretation is explicitly and successfully developed in (Takeuti, [20]). This semantics assumes that elements of a domain are sets (including the empty set which 'corresponds' to empty names). It surely does the job Takeuti claims it does, but we can equivalently use the model-theoretic semantics (developed below) which more emphasizes the fundamental role of individuals.

In the referential-non objectual interpretation the set of existing objects is $D$ anyway. The valuation of name-variables takes subsets of $R$ as values. Nevertheless, the value of propositional expressions does not depend on whether a value of a given name-variable is a non-empty subset of $R \backslash D$, or the empty set. Therefore, as far as we are interested in the truth of an expressions, we may as well accept the model-theoretical interpretation.

\section{I.4 STANDARD SEMANTICS}

We now introduce the standard semantics for BL of Ontology, assuming that the primitive functor is $\varepsilon .7$

A model for $\mathrm{L}^{f}$ is a set $\mathrm{OBJ}$, being a set of objects (interpreted as existing). Let the variables of $L^{f}$ be $\mu_{1}, \ldots, \mu_{n}$, we assume that their order is fixed. $A$ valuation of this variables is a sequence $A^{\mathfrak{u}}=\left\langle A_{1}, \ldots, A_{n}\right\rangle$, where, for every $i, A_{i}$ is a subset of OBJ. Obviously, the value of $\mu_{i}$ in interpretation $A^{u}$ is $A_{i}$. Sometimes, instead of $A_{i}$ as the value of $\mu_{i}$ we shall write $V^{u}\left(\mu_{i}\right)$. For any name constant $\alpha$ its value (a subset of OBJ) is the same for any valuation of name variables. ${ }^{8}$ We shall refer to this value in a given OBJ by ' $\mathrm{V}(\alpha)$ ', where it is clear which OBJ we mean. When it is not important whether $\alpha$ is a name constant or a variable, we refer to its semantic value in a given $O B J$ in a given valuation $u$ of name variables by $\operatorname{EV}^{\mathfrak{u}}(\alpha)$.

\footnotetext{
${ }^{6}$ Of course, one can claim that we cannot use standard set theory in meta-theory of Leśniewski's systems, because Leśniewski did not believe in distributive sets.

I will try to show on an example that mutatis mutandis the same results can be obtain within the Leśniewskian philosophical framework. However, I will not proceed according to this method. In this paper it would only complicate our reasonings.

${ }^{7}$ We might have chosen otherwise. However, it is not an essential decision, as far as all other functors are definable by means of the primitive basis. The semantics for all other functors is to be constructed according to the introduced definitions of these functors.

${ }^{8}$ This account will be modified while discussing the rule of definition in non-standard interpretations.
} 
We define the standard notion of satisfaction: satisfaction ${ }^{\varepsilon}$. It is important that the quantifiers of our meta-language are interpreted differently from the quantifiers of our $L^{f}$. We interpret meta-language individual variables referentially (respective to OBJ) 9

DEFINITION 2 We assume that the sequence of variables is fixed.

I. $\left\ulcorner\mu_{k} \varepsilon \mu_{i}\right\urcorner$ is satisfied ${ }^{\varepsilon}$ in $\mathrm{OBJ}$ by a valuation $\mathrm{A}^{\mathrm{u}}$ if and only if $\exists !_{\chi} \chi \in A_{k} \wedge A_{k} \subseteq$ $A_{i}$.

2. $\ulcorner\neg \chi\urcorner$ is satisfied ${ }^{\varepsilon}$ in $\mathrm{OBJ}$ by the valuation $\mathrm{A}^{u}$ if and only if $\ulcorner\chi\urcorner$ is not satisfied ${ }^{\varepsilon}$ in $\mathrm{OBJ}$ by the valuation $\mathrm{A}^{\mathrm{u}}$.

3. $\left\ulcorner\chi_{i} \wedge \chi_{j}\right\urcorner$ is satisfied ${ }^{\varepsilon}$ in $\mathrm{OBJ}$ by the valuation $A^{\mathfrak{u}}$ if and only if $\left\ulcorner\chi_{i}\right\urcorner$ is satisfied ${ }^{\varepsilon}$ in $\mathrm{OBJ}$ by the valuation $\mathrm{A}^{\mathfrak{u}}$ and $\left\ulcorner\chi_{j}\right\urcorner$ is satisfied ${ }^{\varepsilon}$ in $\mathrm{OBJ}$ by the valuation $\mathrm{A}^{\mathrm{u}}$.

4. $\left\ulcorner\forall_{\mu_{\mathrm{k}}} \chi\right\urcorner$ is satisfied in $^{\varepsilon} \mathrm{OBJ}$ by the valuation $\mathrm{A}^{\mathrm{u}}$ if and only if $\ulcorner\chi\urcorner$ is satisfied in $^{\varepsilon}$ $\mathrm{OBJ}$ by any possible valuation $\mathrm{A}^{\mathrm{d}}$ which is different from $\mathrm{A}^{\mathrm{u}}$ at most at the $\mathrm{k}^{\text {th }}$ place.

A propositional expression is a tautology ${ }^{\varepsilon}$ iff it is satisfied ${ }^{\varepsilon}$ in any domain by any valuation. ${ }^{10}$ In general, if we use upper indices for a given notion of satisfaction, say satisfaction ${ }^{\psi}$, we say that validity ${ }^{\psi}$ consists in being satisfied ${ }^{\psi}$ in any domain by any valuation.

From now on, instead of saying 'an expression $\varphi$ is satisfied ${ }^{\psi}$ in $O B J$ by the valuation $\mathrm{A}^{\delta}$ ' we say: ' $\varphi$ is $\operatorname{SAT}_{\mathrm{OBJ}}^{\psi, \delta}$ '.

\section{I.5 ONTOLOGICAL FUNCTORS}

We conduct a more careful definition of ontological functors elsewhere [2I]. Here, our description will be simplified, but sufficient for our present purpose.

There are some specific functors which we would like to consider in this paper, 2-place Ontological Functors (OF). They are those specific 2-place functors, which distinguish Elementary Ontology from Prototetics. As it is obvious, these functors are of syntactical category $\frac{s}{n, n}$.

However, this information does not suffice for distinguishing 2-place ofs from other functors of the same syntactical category. For example, we would like to consider the expression 'is' in: 'Socrates is mortal.' an of. This functor is of the syntactical category $\frac{\mathrm{s}}{\mathrm{n}, \mathrm{n}}$; but we can as well find a functor of the same syntactical category, which surely is not an of, e.g. the expression 'loves' in 'John loves Mary' is not an of, though it is of the category $\frac{s}{n, n}$.

\footnotetext{
${ }^{9}$ We use the quantifier $\exists$ ! which is to be read: 'there is exactly one'. Of course, it can be defined in terms of the universal quantifier and identity.

${ }^{10}$ In fact, Leśniewski excluded propositional formulae from the set of theorems of his system, so any tautology should be preceded by universal quantifiers binding the otherwise free variables. For our purpose the matter is inessential.
} 
Hence we need some other condition (or a set of conditions), which would not only be necessary, but also sufficient for a 2-place functor to be an of. The simplest intuition seems to be that ofs, concatenated (properly) to their arguments, form expressions concerning the set-theoretical relations between the denotations of their arguments, whereas those functors, which are not OFs, do not. However, the situation is not so simple. We cannot say just that. Still it would remain undecided, what is the nature of this 'concern'.

What we would like first to stipulate, is some kind of extensionality. The value of any proposition built from an of and its arguments should depend on the extensions of these names only. We define the notion of coextensiveness relation, relativized to the valuation $\mathrm{V}^{\mathrm{u}}$ (we denote it in this paper by ${ }^{\text {' }} \dot{\equiv}^{\mathrm{u}}$ ):

DEFINITION $3 \mu_{1} \dot{\doteq}^{\mathrm{u}} \mu_{2} \equiv \mathrm{V}^{\mathrm{u}}\left(\mu_{1}\right)=\mathrm{V}^{\mathrm{u}}\left(\mu_{2}\right)$

Now, we can define the property of extensionality of functors of syntactical category $\frac{s}{n, n} \amalg$,

DEFINITION $4 A$ 2-place functor $\delta_{2}$ is extensional if and only if for all $\mathrm{OBJ}, \mu_{1}, \mu_{2}$, $\mu_{3}, \mu_{4}$, and $u$ : If $\mu_{1} \doteq{ }^{\mathrm{u}} \mu_{2}$ and $\mu_{3} \dot{ }^{\mathrm{u}} \mu_{4}$, then: $\left(\mu_{1} \delta_{2} \mu_{3} \equiv \mu_{2} \delta_{2} \mu_{4}\right)$ is $\mathrm{S}^{\varepsilon}$-satisfied by valuation $\mathrm{V}^{\mathrm{u}}$.

It it is important that the semantic extensionality defined above is something different from the extensionality of the rule of extensionality.

We also shall define the identity of 2-place extensional functors. Two 2place ofs, say $\delta_{2}^{1}, \delta_{2}^{2}$ are identical if and only if the truth conditions of sentences obtained by them and their arguments are the same:

DEFINITION $5 \delta_{2}^{1}=\delta_{2}^{2} \equiv \forall_{\mu_{1}, \mu_{2}}\left[\mu_{1} \delta_{2}^{1} \mu_{2} \equiv \mu_{1} \delta_{2}^{2} \mu_{2}\right]$

The above definitions, mutatis mutandis apply to any k-place functors.

At first, it would perhaps look good to define Ontological Functors as those $k$-place sentence-forming functors of name arguments, which are extensional.

However, it still does not suffice. Let us take the sentence 'John loves Mary.' As we have said, we do not want to count 'loves' as an ontological functor. Now, it is possible to refer uniquely to John by means of other names. For example, his worker, say Frank, refers to him by 'boss'. John's boss refers to him by, say, 'Mr Johnson'. The sentence 'John loves Mary' will not change its value if we replace 'John' by 'Mr Johnson' or 'Frank's boss'. Similarly with all names that uniquely refer to Mary. Hence, we obtain the result that 'loves' is also extensional.

Therefore, we need something more. Once again, how to explicate the 'set theoretical concern' of an ontological functor? We tentatively propose:

\footnotetext{
${ }^{11}$ In introducing this notion we proceed according to the way of defining extensionality in (Borkowski, [2] p.I97-I98]), where he defines extensionality of $\frac{s}{n_{1}, \ldots, n_{k}}$ predicates.
} 
DEFINITION 6 Functor $\mathrm{f}$ is a k-place ontological functor iff it is a sentence-forming functor of $\mathrm{k}$ name-arguments, and $\mathrm{f}$ is definable in metalanguage by expression:

$\mathrm{f}\left(\mu_{1}, \ldots, \mu_{\mathrm{k}}\right)$ is satisfied in OBJ by $\mathrm{A}^{\mathrm{u}}$ iff $\mathrm{X}$

where $\chi$ is a formula of set-theory with $\mu_{1}, \ldots, \mu_{k}$ as the only free variables.

In this paper we shall be mainly concerned with 2-place ofs.

\section{I.6 AXIOMS}

Among axioms of Ontology that were given in the development of Ontology we can distinguish axioms in which the only primitive specific functor of the system is $\varepsilon$, and axioms which either introduce also other functors beside $\varepsilon$, or introduce other functors instead of $\varepsilon$. Let us call the first ' $\varepsilon$-axioms', and the others ' $C$-axioms'.

Historically, Ontology was usually based on a single axiom, however, there are at least a few possibilities of such an axiomatization. Let us list some main known axiomatizations.

\section{I.6.I $\varepsilon$-AXIOMS}

Historically, the following axioms (each one, as a single axiom of Ontology) were suggested (Lejewski, [9. p. I35-136]):

$\operatorname{AXIOM~I~} \forall_{\mathrm{a}, \mathrm{b}}\left[\mathrm{a} \varepsilon \mathrm{b} \equiv\left(\exists_{\mathrm{c}} \mathrm{c} \varepsilon \mathrm{a} \wedge \forall_{\mathrm{c}, \mathrm{d}}(\mathrm{c} \varepsilon \mathrm{a} \wedge \mathrm{d} \varepsilon \mathrm{a} \rightarrow \mathrm{c} \varepsilon \mathrm{d}) \wedge \forall_{\mathrm{c}}(\mathrm{c} \varepsilon \mathrm{a} \rightarrow \mathrm{c} \varepsilon \mathrm{b})\right)\right]$

The axiom I was firstly introduced by Leśniewski in 1920. In I92I he proposed another axiom:

AXIOM $2 \forall_{a, b}\left[a \varepsilon b \equiv\left(\exists_{c}(c \varepsilon a \wedge c \varepsilon b) \wedge \forall_{c, d}(c \varepsilon a \wedge d \varepsilon a \rightarrow c \varepsilon d)\right)\right]$

In the same year Sobociński proved that equivalently we can accept as an axiom:

AXIOM $3 \forall_{a, b}\left[a \varepsilon b \equiv\left(\exists_{c}(c \varepsilon a \wedge c \varepsilon b) \wedge \forall_{c}(c \varepsilon a \rightarrow a \varepsilon c)\right)\right]$

The next simplification was introduced by Leśniewski in 1929:

$\operatorname{AXIOM}_{4} \forall_{a, b}\left(a \varepsilon b \equiv \exists_{c}(a \varepsilon c \wedge c \varepsilon b)\right)$

I.6.2 $\subset$-AXIOMS

Lejewski (Lejewski, [9, p. 137]) introduced an axiom:

$\operatorname{AxIOM} 5 \forall_{\mathrm{a}, \mathrm{b}}\left[\mathrm{a} \bar{\varepsilon} \mathrm{b} \equiv\left(\exists_{\mathrm{c}}(\mathrm{c} \bar{\varepsilon} \mathrm{b} \wedge \neg(\mathrm{c} \bar{\varepsilon} \mathrm{a})) \wedge \forall_{\mathrm{c}, \mathrm{d}, \mathrm{e}}(\mathrm{c} \bar{\varepsilon} \mathrm{d} \wedge \mathrm{d} \bar{\varepsilon} e \rightarrow c \bar{\varepsilon} \mathrm{a} \vee \mathrm{d} \bar{\varepsilon} \mathrm{a})\right)\right]$

where the functor $\varepsilon$ was introduced by the following definition: 
DEFINITION $7 \forall_{\mathrm{a}, \mathrm{b}}\left(\mathrm{a} \varepsilon \mathrm{b} \equiv \exists_{\mathrm{c}}(\mathrm{a} \bar{\varepsilon} \mathrm{c} \wedge \neg(\mathrm{a} \bar{\varepsilon} \mathrm{b}))\right)$

Functor $\bar{\varepsilon}$ is called the functor of singular exclusion. First, on the ground of formulations of Ontology, where $\varepsilon$ was the primitive functor, it was defined:

DEFINITION $8 \forall_{a, b}(a \bar{\varepsilon} b \equiv(a \varepsilon a \wedge \neg(a \varepsilon b)))$

Sobocinski's result tells us that we can accept the functor $<$ as the only primitive one functor of Ontology. This functor originally was introduced by the definition:

DEFINITION $9 \forall_{\mathrm{a}, \mathrm{b}}\left[\mathrm{a}<\mathrm{b} \equiv\left(\exists_{\mathrm{c}} \mathrm{c} \varepsilon \mathrm{a} \wedge \forall_{\mathrm{c}}(\mathrm{c} \varepsilon \mathrm{a} \rightarrow \mathrm{c} \varepsilon \mathrm{b})\right)\right]$

In Sobociński's formulation however, it was the only primitive functor. The axiom was:

AXIOM $6 \forall_{\mathrm{a}, \mathrm{b}}\left[\mathrm{a}<\mathrm{b} \equiv\left(\exists_{\mathrm{c}} \mathrm{c}<\mathrm{a} \wedge \forall_{\mathrm{c}}\left(\mathrm{c}<\mathrm{a} \wedge \forall_{\mathrm{d}}(\mathrm{d}<\mathrm{c} \rightarrow \mathrm{c}<\mathrm{d}) \rightarrow \mathrm{c}<\mathrm{b}\right)\right)\right]$

and the functor $\varepsilon$ was introduced by the definition:

DEFINITION io $\forall_{\mathrm{a}, \mathrm{b}}\left(\mathrm{a} \varepsilon \mathrm{b} \equiv\left(\mathrm{a}<\mathrm{b} \wedge \forall_{\mathrm{c}}(\mathrm{c}<\mathrm{a} \rightarrow \mathrm{a}<\mathrm{c})\right)\right)$

In 1956 Lejewski has shown that we can use $\subset$ as the only primitive functor. In the original formulation it was defined:

DEFINITION II $\forall_{\mathfrak{a}, \mathrm{b}}\left(\mathrm{a} \subset \mathrm{b} \equiv \forall_{\mathfrak{c}}(\mathrm{c} \varepsilon \mathrm{a} \rightarrow \mathfrak{c} \varepsilon b)\right)$

In Lejewski's formulation, $\varepsilon$ was defined:

DEFINITION I2 $\forall_{\mathrm{a}, \mathrm{b}}\left[\mathrm{a} \varepsilon \mathrm{b} \equiv\left(\exists_{\mathrm{c}} \neg(\mathrm{a} \subset \mathrm{c}) \wedge \mathrm{a} \subset \mathrm{b} \wedge\right.\right.$

$$
\left.\left.\forall_{c, d, e}(c \subset a \wedge d \subset a \rightarrow(c \subset d \vee d \subset e))\right)\right]
$$

and Lejewski's axiom (the second, shorter version) was:

AXIOM $7 \forall_{\mathrm{a}, \mathrm{b}}\left[\mathrm{a} \subset \mathrm{b} \equiv \forall_{\mathrm{c}}\left(\mathrm{c} \subset \mathrm{a} \wedge \forall_{\mathrm{d}, \mathrm{e}}(\mathrm{d} \subset \mathrm{c} \rightarrow \mathrm{c} \subset \mathrm{d} \vee \mathrm{d} \subset \mathrm{e}) \rightarrow \mathrm{c} \subset \mathrm{b}\right)\right]$

I.7 RULES

In different formulations of Ontology we can meet different bases of primitive rules of inference. For instance, Luschei [II, p. I4I] enumerates: rule(s) of definition, distribution, detachment, substitution and extensionality. On the other hand, Słupecki [I7, pp. 75-79] equivalently lists: substitution, detachment, omitting the universal quantifier, omitting the particular quantifier ${ }^{\mathrm{I2}}$, adding the universal quantifier, adding the particular quantifier, the rule of definition, and the rule of extensionality.

In our investigations we will be interested whether the above mentioned rules are validity-preserving in non-standard interpretations of primitive functors. In the presentation of rules, we shall follow the Słupecki's account.

\footnotetext{
${ }^{\mathrm{I}}$ Słupecki calls it 'existential'. For the reasons already given, we choose another name.
} 
RULE OF SUbSTITUtion The original Leśniewski's account of this rule is a little bit foreign to the modern logical language. His description of this rule uses numerous technical terms of his own. We'll try to give a simplified formulation of this rule, which (when given with the rules of adding and omitting quantifiers) does the same job as the original one ${ }^{13}$

Let $\chi$ be a propositional expression with at least one free variable $v$ of syntactic category $\sigma$. From $\chi$ infer an expression $\chi_{1}$ which differs from $\chi$ in the following aspects:

I. Instead of each free occurrence of $v$ in $\chi$ there is an expression (simple or not) $\mu$ of the category $\sigma$.

2. It is not the case that there is in $\mu$ a free variable $v$ and $v$ occurs in $\chi$ as a free variable within a range of a quantifier binding $v$.

In other words, we substitute for each free occurrence of a variable so that no variable becomes bound.

THEOREM I The rule of substitution is validity-preserving in $\mathrm{BL}$, independently of the choice of the interpretation of a primitive functor.

PROOF: In BL the only variables which can be substituted for are name variables. They can be substituted either by name variables or by name constants, as far as the latter have been introduced by means of the rule of definition.

The semantics for name variables has been introduced. Semantics of name constants is very intuitive: they denote for each OBJ a fixed subset of a domain, and this denotation does not depend on the interpretation of variables.

Now assume that a given expression $\chi$ of BL is valid and $v$ is a free name variable in $\chi$. Then, obviously, $\chi$ is satisfied in any model; in any domain and in any valuation, particularly in any interpretation of $v$.

${ }^{13}$ Let's take the Luschei's translation of this description [II] pp. 252-253]. It has I4 points. For the sake of example, I quote the 3 rd and 9 th:

(3) For every E and F, if E is d, F is a word inside the subquantifier of C, and there are exactly as many $\mathrm{d}$ that precede $\mathrm{E}$ as words inside the subquantifier of $\mathrm{C}$ that precede $\mathrm{F}$, then, for at least one $\mathrm{G}, \mathrm{F}$ is a variable of $\mathrm{C}$ bound by $\mathrm{G}$ or is an expression equiform to $\mathrm{E}$.

(9) For every E, if E is a term in A, then either (i) E is an argument or functor of an ingredient of $\mathrm{A}$ and suited to be constant, relative to thesis $\mathrm{B}$ of this system;

(ii) for at least one $\mathrm{F}, \mathrm{E}$ is a word inside $\mathrm{F}$, and $\mathrm{F}$ is a universal quantifier in $\mathrm{A}$; or

(iii) for certain $\mathrm{F}$ and $\mathrm{G}, \mathrm{F}$ is in $\mathrm{A}$, and $\mathrm{E}$ is a variable of generalization $\mathrm{F}$ bound by G.

Clearly, a rule given in 44 points of similar style is not user-friendly. 
Now, let $\chi_{1}$ be a result of replacing $v$ in $\chi$ by $\mu$. Since $\chi_{1}$ differs from $\chi$ only in the form of one free variable, the conditions of validity of $\chi_{1}$ differ only in the fact that we talk about all possible interpretations of $\mu$ instead of that of $v$. In any model, possible different interpretations of $\mu$ are exactly the same as possible interpretations of $v$ - namely all subsets of a model's domain. Hence, $\chi_{1}$ is also valid.

In the case of substituting a name variable by a name constant, the case is even simpler. In general, if a formula is valid in any possible interpretation of a given variable $v$, in fact, it yields a valid result if we admit only one interpretation of this variable (the intended denotation of the name we want to substitute). Q.E.D.

REMARK It is interesting, how the above theorem can be extended to the full language of Ontology. Let us sketch a possible and quite a simple way of doing so, on the example of variables and constants of the category $\frac{s}{n, n}$.

If we want to consider variables and constants of this category, it will be convenient to introduce their 'denotation' or 'valuation'. Name constants have some fixed subsets of $\mathrm{OBJ}$ as denotations. Name variables have subsets of $\mathrm{OBJ}$ as possible valuations. Accordingly, intuitive candidates for our job will be binary relations between the subsets of OBJ. Strictly speaking, not all possible relations, but only the relations corresponding to definientes of possible definitions of ontological functors, when a semantic interpretation of the primitive basis is given $\sqrt{14}$

For example, the relation corresponding to the standard interpretation of $\varepsilon$ is the relation that takes place between two subsets of a given OBJ iff the first is a singleton and a subset of the second. Relations corresponding to other, non-primitive functors are to be settled in accordance with their definitions in the system.

Clearly, OF-constants in a given model OBJ have fixed relations in $2^{\mathrm{OBJ}}$ as semantic correlates. Valuations of of-variables of a given language in a given model will be sequences of (admissible) relations in $2^{\mathrm{OBJ}}$. Semantic interpretation of quantifiers binding OF-variables is intuitive - it repeats the schema for name variables, differing only in the set of admissible valuations of ofvariables. Now, the proof that the rule of substitution for such variables is validity-preserving should work in a similar manner as that for name-variables.

Full language of Ontology is not restricted only to these two kinds of variables and constants. It admits variables of any category built from n-s or s-s and any constants of any admissible category, if these constants are introduced by properly constructed definitions.

It seems that mutatis mutandis we can extend our semantics to let it account for any admissible category (perhaps it would be then convenient to introduce

\footnotetext{
${ }^{14}$ For details concerning possible definitions of ontological functors etc. see (Urbaniak, [2I]).
} 
explicitly 1 and 0 as semantic correlates of propositions). These are only technical details 15 Hence:

НY РОтНЕSIS I The rule of substitution is validity-preserving in the full language of Ontology.

RULES FOR QUANTIFICATION The rules governing the use of quantifiers are very similar to that occurring in systems of natural deduction - for instance, in the formulation introduced in (Borkowski, [2]).

Their validity ${ }^{16}$ is based, freely speaking, on the relations between quantification, free variables and semantic correlates of expressions of a given category, just as it is in first- or second-order predicate logic. Interpretation of of-constants does not interfere with their validity, hence:

HY РОTHESIS 2 Rules governing the use of quantifiers are validity-preserving independently on the interpretation of $\mathrm{OF}-\mathrm{constants}$.

Now, we will simply present the rules of definition and of extensionality. Later on, we shall try to answer the question whether these rules preserve validity in given non-standard interpretations.

RULE OF EXTENSIONALITY This rule is an axiomatic rule introducing implications of a specific form (so called lawe of extensionality) to a system irrespective of what theorems were subjoined thereto before. In its presentation we shall follow (Słupecki, [17, p. IOI]).

Let the variable $\chi$ represent propositional function (complex or simple - what is important is the category of $\chi$ and not its complexity) of one argument, and let the not equiform variables $\lambda$ and $\delta$ be admissible arguments of this function. Let $\chi(\lambda)$ and $\chi(\delta)$ be applications of $\chi$ to, respectively, $\lambda$ and $\delta$. The consequents of the laws of extensionality have the form:

$$
\forall_{\chi}[\chi(\lambda) \equiv \chi(\delta)]
$$

The form of the antecedent depends on the category of $\lambda$ and $\delta$. If they are nominal variables not having the form of the variable $x$, the antecedent has the form:

$$
\forall_{x}[x \varepsilon \lambda \equiv x \varepsilon \delta]
$$

\footnotetext{
${ }^{15}$ Though it is interesting question, how to construct quite a simple semantics for the full language of Ontology, which (semantics) would not only do its formal job, but also would work in accordance with Leśniewski's philosophical commitment, i.e. would not introduce other entities than individuals.

${ }^{16}$ When there is no danger of ambiguity, we use 'validity' with reference to rules as a name of the property of being validity-preserving.
} 
If $\lambda$ and $\delta$ are functors of $k$ arguments, and $\alpha_{1}, \ldots, \alpha_{k}$ are (i) arguments of both $\lambda$ and $\delta$, (ii) none two of them are equiform, and (iii) none has the form of the variable $x$, then we consider two possibilities.

I. Both $\lambda$ and $\delta$ are name-formative. Then, the antecedent has the form:

$$
\forall x, \alpha_{1}, \ldots, \alpha_{k}\left[x \varepsilon \lambda\left(\alpha_{1}, \ldots, \alpha_{k}\right) \equiv x \varepsilon \delta\left(\alpha_{1}, \ldots, \alpha_{k}\right)\right]
$$

2. Both $\lambda$ and $\delta$ are proposition-formative. Then, the antecedent has the form:

$$
\forall \alpha_{1}, \ldots, \alpha_{k}\left[\lambda\left(\alpha_{1}, \ldots, \alpha_{k}\right) \equiv \delta\left(\alpha_{1}, \ldots, \alpha_{k}\right)\right]
$$

RULE OF DEFINition We shall first present this rule for nominal constants and name-formative or proposition-formative functors of nominal arguments according to (Słupecki, [I7, pp. 74-75]), and then extend it to the full language of Ontology, following (Słupecki, [17, pp. IOO-IOI]).

I. The schema for defining a nominal constant, say $\alpha$, is as follows:

$$
\forall_{\mathrm{b}}(\mathrm{b} \varepsilon \alpha \equiv \mathrm{b} \varepsilon \mathrm{b} \wedge \chi)
$$

where $\chi$ is a propositional expression of $\mathrm{BL}$.

2. The schema for a definition of a proposition-formative functor, say $\lambda$, of nominal arguments is:

$$
\lambda\left(b_{1}, \ldots, b_{n}\right) \equiv \chi
$$

where $\chi$ is as before.

3. The schema for defining a name-formative functor, say $\delta$, is as follows:

$$
b \varepsilon \delta\left(b_{1}, \ldots, b_{n}\right) \equiv b \varepsilon b_{i} \wedge \chi
$$

where $0 \leqslant i \leqslant n$ and $\chi$ is as above.

In definitions other than these above only functors are defined. These functors are either proposition-formative or name-formative. The forms of definitions do not differ essentially from the above schemata, but the functors of the full language of Ontology may depend on parameters. These parameters are placed inside parentheses differing in shape from those enclosing arguments of functors. For every variable acting as a parameter of a functor, the 
definiens of the definition of this functor has to include a free variable equiform to that occurring in the parameter, and every variable acting as a parameter differs in form from all other variables appearing in the definiendum. The rule of substitution may affect parameters as well.

This rule, even for BL, is a cause of problems which throw some light on some interesting issues, which will be discussed later on.

\section{ADHERENTS OF STANDARD SEMANTICS}

The Standard Semantics of Ontology consists in accepting the satisfaction ${ }^{\varepsilon}$ as the notion of satisfaction for the $\varepsilon$ functor. This interpretation of $\varepsilon$ consists in the following underlying intuition:

Let $a, b$ be names. The expression $a \varepsilon b$ is true if and only if $a$ is an unshared name (i. e. having exactly one designate) and, either $b$ is an unshared name of the object named by $a$, or $b$ is a shared name (i. e. having more than one designate) naming also the object named by a.

This interpretation is accepted by numerous logicians $\sqrt{7}$

What is more interesting, almost all of them explicitly claim that this meaning of $\varepsilon$ functor is determined by the Axiom II (Slupecki, [I7, p. 72-73], Lejewski, [9, p. I35-I36], Hiż, [4, p. 273]); perhaps (Iwanuś, [5, p. I68-I69]) is the most exemplary:

The only primitive term of ontology is the constant ' $\varepsilon$ ', its meaning is determined by the following axiom ...

similarly, (Lejewski, [IO, p. 323]):

...the meaning of the copula 'is' (' $\varepsilon$ ' in symbols) is determined axiomatically ...

What does it mean that an axiom determines the meaning of the primitive constant? Such a claim is an equivalence, which states that

I. If we accept the 'determined' meaning of the constant, the axiom is valid.

2. If the axiom is valid, then the constant has the such 'determined' meaning.

${ }^{17}$ E.g.: (Borkowski, [2] p. I88]), (Sobociński, [19 p. I4]), (Lejewski, [8, p. 54-55]), (Lejewski, [9. p. I29]), (Słupecki, [I7, p. 65-66]), (Canty, [3] p. I49-15I]). 
In the first point the mentioned adherents are right, in the sense, that in the Standard Semantic the Axiom 国 (and other axioms) are valid ${ }^{\varepsilon}$ in the standard interpretation of $\varepsilon$.

Our purpose is to show that they are wrong in the second claim - we shall argue that we can understand $\varepsilon$ in at least $\boldsymbol{x}_{0}$ different ways and keep axioms valid in this interpretations.

We must also point out that it is not clear, whether the expression 'determined by an axiom' means the same as 'determined axiomatically'. The second expression can be quite legitimately interpreted as 'determined by axiomatic basis, i.e. by axiom(s) and rule(s) of a given system'. In the second sense, we must also consider additional two conditions.

I. If we attribute to a constant the standard interpretation, all rules preserve validity.

2. If the validity of rules is to be preserved, the constant has to have the standard interpretation.

In other words, for a given axiomatic basis and for any non-standard interpretation we will be interested not in one, but in two questions. First, whether its axiom is valid in this interpretation of the primitive constant, and second, whether all rules preserve validity in this interpretation of this constant ${ }_{18}^{8}$

Adherents of the uniqueness of the admissible interpretation of $\varepsilon$ can remain unconvinced when they are shown a non-standard interpretation which preserves axiom(s). However, non-standard interpretations which not only keep axiom(s) valid, but also make rules validity-preserving, may be for them really troublesome.

In this paper we shall not only show that there are non-standard interpretations which keep axiom(s) safe (it is our first purpose), but also we shall discuss, whether in these interpretations rules of Ontology are validity-preserving.

\section{NON-STANDARD VALIDITY OF AXIOMS}

\section{I Satisfaction ${ }^{\Phi}$ AND $\varepsilon-A x i O M S$}

Let us instead of satsifaction ${ }^{\varepsilon}$ accept the following definition of satisfaction:

DEFINITION I3 We assume that the sequence of variables is fixed.

I. $\left\ulcorner\mu_{\mathrm{k}} \varepsilon \mu_{i}\right\urcorner$ is satisfied ${ }^{\Phi}$ in $\mathrm{OBJ}$ by a valuation $\mathrm{A}^{\mathrm{u}}$ if and only if $\mathrm{A}_{\mathrm{k}}=\mathrm{A}_{i}=\Phi$.

2. $\ulcorner\neg \chi\urcorner$ is satisfied $\Phi^{\Phi}$ in $\mathrm{OBJ}$ by the valuation $\mathrm{A}^{\mathrm{u}}$ if and only if $\ulcorner\chi\urcorner$ is not satisfied ${ }^{\Phi}$ in $\mathrm{OBJ}$ by the valuation $\mathrm{A}^{\mathrm{u}}$.

${ }^{18}$ Note that, though we discuss soundness in non-standard interpretations. It is an interesting question which we shall not discuss in this paper, whether Ontology is a sound and complete theory in some non-standard interpretation. 
3. $\left\ulcorner\chi_{i} \wedge \chi_{j}\right\urcorner$ is satisfied ${ }^{\Phi}$ in $\mathrm{OBJ}$ by the valuation $\mathrm{A}^{\mathrm{u}}$ if and only if $\left\ulcorner\mathrm{\chi}_{i}\right\urcorner$ is satisfied ${ }^{\Phi}$ in $\mathrm{OBJ}$ by the valuation $\mathrm{A}^{\mathrm{u}}$ and $\left\ulcorner\chi_{\mathfrak{j}}\right\urcorner$ is satisfied ${ }^{\Phi}$ in $\mathrm{OBJ}$ by the valuation $\mathrm{A}^{\mathrm{u}}$.

4. $\left\ulcorner\forall_{\mu_{k}} \chi\right\urcorner$ is satisfied ${ }^{\Phi}$ in $\mathrm{OBJ}$ by the valuation $A^{\mathcal{u}}$ if and only if $\ulcorner\chi\urcorner$ is satisfied ${ }^{\Phi}$ in $\mathrm{OBJ}$ by any possible valuation $\mathrm{A}^{\mathrm{d}}$ which is different from $\mathrm{A}^{\mathrm{u}}$ at most at the $k^{\text {th }}$ place.

As an obvious consequence of the standard definition of particular quantifier, we may add:

$\left\ulcorner\exists_{\mu_{k}} \chi\right\urcorner$ is satisfied ${ }^{\Phi}$ in OBJ by a valuation $A^{u}$ if and only if $\ulcorner\chi\urcorner$ is satisfied ${ }^{\Phi}$ in OBJ by some possible valuation $A^{\mathrm{d}}$ which is different from $A^{u}$ at most at the $k^{\text {th }}$ place.

TheOrem 2 Axiom Iis valid ${ }^{\Phi}$.

PROOF: Implication to the right: We assume that $a \varepsilon b$ is $S A T_{\mathrm{OBJ}}^{\Phi} \mathrm{u}$. It is to be proven, that $\exists_{\mathrm{c}} c \varepsilon a \wedge \forall_{\mathrm{c}, \mathrm{d}}(\mathrm{c} \varepsilon \mathrm{a} \wedge \mathrm{d} \varepsilon \mathrm{a} \rightarrow \mathrm{c} \varepsilon \mathrm{d}) \wedge \forall_{\mathrm{c}}(\mathrm{c} \varepsilon \mathrm{a} \rightarrow \mathrm{c} \varepsilon b)$ is $\operatorname{SAT}_{\mathrm{OBJ}}^{\Phi} \mathrm{u}$. Since $a \varepsilon b$ is $S_{A} T_{O B J}^{\Phi, ~}, V^{u}(a)=V^{u}(b)=\Phi$. There is a valuation $A^{s}$ which differs from $A^{\mathfrak{u}}$ at most in the fact that the $V^{s}(\mathrm{c})=\Phi$ such that $c \varepsilon a$ is SAT $T_{O B J}^{\Phi, s}$. Hence, $\exists_{c} c \varepsilon a$ is $S A T_{O B J}^{\Phi, u}$.

Now we shall show that with this assumptions

$$
\forall_{c, d}(c \varepsilon a \wedge d \varepsilon a \rightarrow c \varepsilon d)
$$

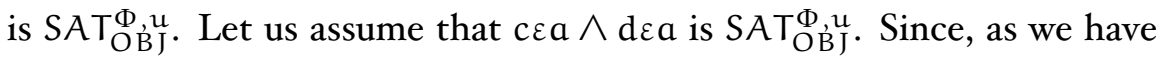
said, $\mathrm{V}^{\mathfrak{u}}(\mathrm{a})=\Phi$, it must be the case that also $\mathrm{V}^{\mathrm{u}}(\mathrm{c})=\mathrm{V}^{\mathrm{u}}(\mathrm{d})=\Phi$. If this is the case, also $c \varepsilon d$ is $S A T_{O B J}^{\Phi, u}$.

It remains to show that on the ground of our assumptions,

$$
\forall \mathrm{c}(\mathrm{c} \varepsilon \mathrm{a} \rightarrow \mathrm{c} \varepsilon \mathrm{b})
$$

is $\operatorname{SAT}_{\mathrm{OBJ}}^{\Phi}$. To do so, assume that $c \varepsilon a$ is $\operatorname{SAT}_{\mathrm{OBJ}}^{\Phi, \mathrm{u}}$. Then, obviously, $\mathrm{V}^{\mathfrak{u}}(\mathrm{c})=\mathrm{V}^{\mathfrak{u}}(\mathrm{a})=\Phi$. Since, according to our previous assumption, $\mathrm{V}^{\mathfrak{u}}(\mathrm{b})=\Phi$, we obtain $\mathrm{V}^{\mathfrak{u}}(\mathrm{c})=\mathrm{V}^{\mathfrak{u}}(\mathrm{b})=\Phi$. It follows that $c \varepsilon b$ is $S A T_{O B J}^{\Phi} \mathrm{u}$, what ends the proof in one direction.

Implication to the left: We assume that

$$
\exists_{\mathrm{c}} \mathfrak{c} \varepsilon \mathrm{a} \wedge \forall_{\mathrm{c}, \mathrm{d}}(\mathrm{c} \varepsilon \mathrm{a} \wedge \mathrm{d} \varepsilon \mathrm{a} \rightarrow \mathrm{c} \varepsilon \mathrm{d}) \wedge \forall_{\mathrm{c}}(\mathrm{c} \varepsilon \mathrm{a} \rightarrow \mathrm{c} \varepsilon \mathrm{b})
$$

is $S A T_{O B J}^{\Phi, u}$, and obtain in consequence that $a \varepsilon b$ is $S A T_{O B J}^{\Phi, u}$.

Since $\exists_{\mathfrak{c}} c \varepsilon a$, there is a valuation $A^{s}$ differing at most in the value assigned to $c$ such that $c \varepsilon a$ is $S A T_{\mathrm{OBJ}}^{\Phi, s}$. Therefore, $\mathrm{V}^{s}(\mathrm{c})=\mathrm{V}^{\mathrm{s}}(\mathrm{a})=\Phi$ Since $A^{s}$ differs from $A^{\mathfrak{u}}$ only in $V^{s}(c)$, it is the case that $V^{\mathfrak{u}}(a)=\Phi$. Let us 
now notice that $\forall_{c}(c \varepsilon a \rightarrow c \varepsilon b)$ is $\operatorname{SAT}_{\mathrm{OB} \mathrm{J}}^{\Phi}$. As we have said, there is a valuation $A^{s}$ differing at most in the value assigned to $c$ such that $c \varepsilon a$ is $S A T_{\mathrm{OBJ}}^{\Phi, s}$. Hence, $c \varepsilon b$ is $\operatorname{SAT}_{\mathrm{OBJ}}^{\Phi, \mathrm{s}}$. It means that $\mathrm{V}^{\mathrm{s}}(\mathrm{c})=\mathrm{V}^{\mathrm{s}}(\mathrm{b})=\Phi$. But $A^{s}$ differs from $A^{\mathfrak{u}}$ at most in the value assigned to $c$. Therefore $\mathrm{V}^{\mathrm{s}}(\mathrm{b})=\mathrm{V}^{\mathfrak{u}}(\mathrm{b})=\Phi$. Hence $\mathrm{V}^{\mathfrak{u}}(\mathrm{a})=\mathrm{V}^{\mathfrak{u}}(\mathrm{b})=\Phi$. It means that $\mathrm{a} \varepsilon \mathrm{b}$ is $S A T_{O B J}^{\Phi, u}$, what ends the proof (if we take under consideration that we can repeat our proof for any $O B J$ and any valuation). Q.E.D.

The proof given above may be stated in a more intuitive, but less precise and not quite exact manner.

Implication to the right: Assume $a \varepsilon b$. Hence, both $a$ and $b$ are empty names. Therefore, $b \varepsilon a$. If it is so, then also $\exists_{c} c \varepsilon a$. Now assume $c \varepsilon a \wedge d \varepsilon a$. then, both $c$ and $d$ are empty. Hence $c \varepsilon d$. Therefore, $\forall_{c, d}(c \varepsilon a \wedge d \varepsilon a \rightarrow c \varepsilon d)$. Assume

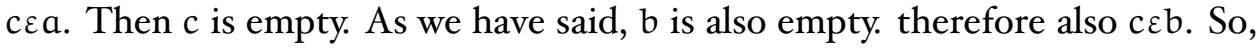
$\forall_{\mathrm{c}}(\mathrm{c} \varepsilon \mathrm{a} \rightarrow \mathrm{c} \varepsilon \mathrm{b})$.

Implication to the left: Assume

$$
\exists_{\mathrm{c}} \mathrm{c} \varepsilon \mathrm{a} \wedge \forall_{\mathrm{c}, \mathrm{d}}(\mathrm{c} \varepsilon \mathrm{a} \wedge \mathrm{d} \varepsilon \mathrm{a} \rightarrow \mathrm{c} \varepsilon \mathrm{d}) \wedge \forall_{\mathrm{c}}(\mathrm{c} \varepsilon \mathrm{a} \rightarrow \mathrm{c} \varepsilon \mathrm{b})
$$

If $\exists_{\mathrm{c}} \mathcal{C} \mathrm{a}$, it means that some name is empty together with a. So a is empty. Since $\forall_{c}(c \varepsilon a \rightarrow c \varepsilon b)$, every name which is empty together with a is empty together with $b$. If $a$ is empty together with itself, $a$ is empty together with $b$. In fact, aea; hence $a \varepsilon b$, which ends the proof.

What we would like to show is that it does not matter which axiom we choose, our non-standard interpretation of $\varepsilon$ makes this axiom valid.

One could argue that we do not have to prove it independently for each axiom. It has been already shown that those axioms are on the ground of Ontology equivalent, so the first proof suffice for our purpose. Nevertheless, we would like to obtain our results independently of the results concerning rules of Ontology.

TheOrem 3 Axiom 2 is valid $^{\Phi}$.

PROOF: Implication to the right: Assume that $a \varepsilon b$ is $\operatorname{SAT}_{\mathrm{OB} \mathrm{J}}^{\Phi}$. Then, $\mathrm{V}^{\mathrm{u}}(\mathrm{a})=$ $\mathrm{V}^{\mathfrak{u}}(\mathrm{b})=\Phi$. There is the valuation $A^{s}$ which differs from $A^{\mathfrak{u}}$ at most in the fact that $V^{s}(c)=\Phi$ such that the formula $c \varepsilon a \wedge c \varepsilon b$ is $S A T_{O B J}^{\Phi}$, Since $A^{u}$ differs from $A^{s}$ only in the valuation of $c, \exists_{c}(c \varepsilon a \wedge c \varepsilon b)$ is $\operatorname{SAT}_{\mathrm{OBJ}}^{\Phi} \mathrm{u}$.

Let us now assume that $c \varepsilon a \wedge d \varepsilon a$ is $\operatorname{SAT}_{\mathrm{OBJ}}^{\Phi}$. This means that $\mathrm{V}^{\mathrm{u}}(\mathrm{c})=$ $\mathrm{V}^{\mathfrak{u}}(\mathrm{a})=\mathrm{V}^{\mathfrak{u}}(\mathrm{d})=\Phi$. Obviously, then $\mathrm{c} \varepsilon \mathrm{d}$ is $S A T_{O B \mathrm{~J}}^{\Phi}$.

Therefore, $\forall_{c, d}(c \varepsilon a \wedge d \varepsilon a \rightarrow c \varepsilon d)$ is $\operatorname{SAT}_{\mathrm{OBJ}}^{\Phi} \mathrm{u}$.

Implication to the left: Assume $\exists_{\mathrm{c}}(\mathrm{c} \varepsilon \mathrm{a} \wedge \mathrm{c} \varepsilon \mathrm{b}) \wedge \forall_{\mathrm{c}, \mathrm{d}}(\mathrm{c} \varepsilon \mathrm{a} \wedge \mathrm{d} \varepsilon \mathrm{a} \rightarrow \mathrm{c \varepsilon d})$ is $S A T_{\mathrm{OBJ}}^{\Phi}$. If $\exists_{\mathrm{c}}(c \varepsilon a \wedge c \varepsilon b)$ is $S A T_{\mathrm{OB} J}^{\Phi, \mathrm{u}}$, then there is a valuation $A^{s}$ differing 
from $A^{u}$ at most in the value assigned to $c$ such that $c \varepsilon a \wedge c \varepsilon b$ is $S A T_{O B J}^{\Phi, s}$. Therefore, $\mathrm{V}^{\mathrm{s}}(\mathrm{c})=\mathrm{V}^{\mathrm{s}}(\mathrm{a})=\mathrm{V}^{\mathrm{s}}(\mathrm{b})=\mathrm{V}^{\mathfrak{u}}(\mathrm{a})=\mathrm{V}^{\mathfrak{u}}(\mathrm{b})=\Phi$. Hence, $\mathrm{a} \varepsilon \mathrm{b}$ is $S A T_{\mathrm{OBJ}}^{\Phi, \mathrm{u}}$, what (since we can reason in the same way for any model and any valuation) ends the proof. Q.E.D.

Of course, there is also the 'light' version of this reasoning.

Implication to the right: Assume $a \varepsilon b$. Then $a$ and $b$ are empty. Since, $a$ is empty together with itself, $a \varepsilon a$. Hence, $a \varepsilon a \wedge a \varepsilon b$. Therefore, $\exists_{c}(c \varepsilon a \wedge c \varepsilon b)$.

Now assume $c \varepsilon a \wedge d \varepsilon a$. Then, obviously, $a, c, d$ are together empty. Hence ced.

Implication to the left: Assume

$$
\exists_{\mathrm{c}}(\mathrm{c} \varepsilon \mathrm{a} \wedge \mathrm{c} \varepsilon \mathrm{b}) \wedge \forall_{\mathrm{c}, \mathrm{d}}(\mathrm{c} \varepsilon \mathrm{a} \wedge \mathrm{d} \varepsilon \mathrm{a} \rightarrow \mathrm{c} \varepsilon \mathrm{d})
$$

Since $\exists_{\mathfrak{c}}(c \varepsilon a \wedge c \varepsilon b)$ there is a name which is together empty with $a$ and $b$. Therefore, $a$ and $b$ are both empty. Hence $a \varepsilon b$, what ends the reasoning.

THEOREM 4 Axiom 3 is valid $^{\Phi}$.

PROOF: Implication to the right: Assume $a \varepsilon b$ is $\operatorname{SAT}_{\mathrm{OBJ}}^{\Phi, \mathrm{u}}$. Hence $\mathrm{V}^{\mathrm{u}}(\mathrm{a})=$ $\mathrm{V}^{\mathfrak{u}}(\mathrm{b})=\Phi$. There is a valuation $A^{s}$ which differs from $A^{\mathfrak{u}}$ at most in the fact that $V^{s}(c)=\Phi$. The formula $c \varepsilon a \wedge c \varepsilon b$ is $\operatorname{SAT}_{O T^{\prime} \mathrm{s}}^{\Phi}$. Since $A^{u}$ differs from $A^{s}$ only in the valuation of $c, \exists_{c}(c \varepsilon a \wedge c \varepsilon b)$ is $S A T_{O B}^{\Phi, u}$.

Assume moreover that for some $k, c \varepsilon a$ is $\operatorname{SAT}_{\mathrm{OBJ}}^{\Phi}$. It means that $\mathrm{V}^{k}(\mathrm{c})=$ $\mathrm{V}^{\mathrm{k}}(\mathrm{a})=\Phi$. It is obvious that then $a \varepsilon c$ is $\operatorname{SAT}_{\mathrm{OBJ}}^{\Phi, k}$.

Hence $\forall_{c}(c \varepsilon a \rightarrow a \varepsilon c)$ is $\operatorname{SAT}_{\mathrm{OBJ}}^{\Phi, \psi}$.

Implication to the left: Assume $\exists_{\mathrm{c}}(\mathrm{c} \varepsilon \mathrm{a} \wedge \mathrm{c} \varepsilon b) \wedge \forall_{\mathrm{c}}(\mathrm{c} \varepsilon \mathrm{a} \rightarrow \mathrm{a} \varepsilon \mathrm{c})$ is $S A T_{\mathrm{OB}}^{\Phi} \mathrm{u}$. If $\exists_{c}(c \varepsilon a \wedge c \varepsilon b)$ is $S A T_{O B J}^{\Phi, u}$, then there is a valuation $A^{s}$ differing from $A^{u}$ at most in the value assigned to $c$ such that $c \varepsilon a \wedge c \varepsilon b$ is $\operatorname{SAT}_{\mathrm{OB}}^{\Phi}, \mathrm{s}$. Therefore, $\mathrm{V}^{\mathrm{s}}(\mathrm{c})=\mathrm{V}^{\mathrm{s}}(\mathrm{a})=\mathrm{V}^{\mathrm{s}}(\mathrm{b})=\mathrm{V}^{\mathfrak{u}}(\mathrm{a})=\mathrm{V}^{\mathfrak{u}}(\mathrm{b})=\Phi$. Hence, $\mathrm{a} \varepsilon \mathrm{b}$ is $S A T_{\mathrm{OBJ}}^{\Phi}, \mathrm{u}$, what (since we can reason in the same way for any model and any valuation) ends the proof. Q.E.D.

Theorem 5 Axiom 4 is valid ${ }^{\Phi}$.

Proof: Implication to the right: Assume that $a \varepsilon b$ is $\operatorname{SAT}_{\mathrm{OBJ}}^{\Phi, \mathrm{u}}$. Hence $\mathrm{V}^{\mathrm{u}}(\mathrm{a})=$ $\mathrm{V}^{\mathfrak{u}}(\mathrm{b})=\Phi$. There is a valuation $A^{\mathrm{s}}$ which differs from $A^{\mathfrak{u}}$ at most in the fact that $V^{s}(c)=\Phi$. The formula $a \varepsilon c \wedge c \varepsilon b$ is $S A T_{O B J}^{\Phi}, s$. Since $A^{u}$ differs from $A^{s}$ only in the valuation of $c, \exists_{c}(a \varepsilon c \wedge c \varepsilon b)$ is $S A T_{O B J}^{\Phi}$.

Implication to the left: Assume that $\exists_{\mathrm{c}}(a \varepsilon c \wedge c \varepsilon b)$ is $S A T_{\mathrm{OBJ}}^{\Phi, \mathrm{u}}$. Hence, there is a valuation $A^{s}$ which differs from $A^{u}$ at most in the fact that $V^{s}(c)=\Phi$, such that $a \varepsilon c \wedge c \varepsilon b$ is $S A T_{O B J}^{\Phi}$. If it is so, $V^{s}(a)=V^{s}(c)=V^{s}(b)=\Phi$ 
Since, as we have said, $A^{s}$ differs from $A^{u}$ at most in the fact that $V^{s}(c)=$ $\Phi$, it is also the case that $\mathrm{V}^{\mathfrak{u}}(\mathrm{a})=\mathrm{V}^{\mathfrak{u}}(\mathrm{b})=\Phi$. Therefore, $a \varepsilon b$ is $S A T_{O B}^{\Phi} \mathrm{u}$. We can repeat this reasoning for any $O B J$ and any $A^{u}$, what ends the proof. Q.E.D.

\subsection{SATISFACTION ${ }^{\mathrm{F}}$ AND $\varepsilon$-AXIOMS}

Let us instead of satsifaction ${ }^{\Phi}$ interpret $\varepsilon$ as falsum functor, which always yields $O$ as value for any arguments. Therefore we accept the following definition of satisfaction ${ }^{\mathrm{F}}$ :

DEFINITION I4 We assume that the sequence of variables is fixed.

I. $\left\ulcorner\mu_{\mathrm{k}} \varepsilon \mu_{\mathrm{i}}\right\urcorner$ is satisfied ${ }^{\mathrm{F}}$ in $\mathrm{OBJ}$ by a valuation $\mathrm{A}^{\mathrm{u}}$ if and only if $A_{k} \neq A_{k}$.

2. $\ulcorner\neg \chi\urcorner$ is satisfied $d^{\mp}$ in $\mathrm{OBJ}$ by the valuation $\mathrm{A}^{\mathrm{u}}$ if and only if $\ulcorner\chi\urcorner$ is not satisfied ${ }^{\mathrm{F}}$ in $\mathrm{OBJ}$ by the valuation $\mathrm{A}^{\mathrm{u}}$.

3. $\left\ulcorner\chi_{i} \wedge \chi_{j}\right\urcorner$ is satisfied ${ }^{\mp}$ in $\mathrm{OBJ}$ by the valuation $A^{u}$ if and only if $\left\ulcorner\chi_{i}\right\urcorner$ is satisfied ${ }^{F}$ in $\mathrm{OBJ}$ by the valuation $\mathrm{A}^{\mathrm{u}}$ and $\left\ulcorner\chi_{\mathrm{j}}\right\urcorner$ is satisfied ${ }^{\mathrm{F}}$ in $\mathrm{OBJ}$ by the valuation $\mathrm{A}^{\mathrm{u}}$.

4. $\left\ulcorner\forall_{\mu_{\mathrm{k}}} \chi\right\urcorner$ is satisfied $\mathrm{F}^{\mathrm{F}}$ in $\mathrm{OBJ}$ by the valuation $A^{\mathrm{u}}$ if and only if $\ulcorner\chi\urcorner$ is satisfied ${ }^{\mathrm{F}}$ in $\mathrm{OBJ}$ by any possible valuation $\mathrm{A}^{\mathrm{d}}$ which is different from $\mathrm{A}^{\mathrm{u}}$ at most on one place, namely $\mathrm{k}$ - th (place).

As an obvious consequence of the standard definition of particular quantifier, we may add:

$\left\ulcorner\exists_{\mu_{k}} \chi\right\urcorner$ is satisfied ${ }^{\mathrm{F}}$ in OBJ by the valuation $A^{u}$ if and only if $\ulcorner\chi\urcorner$ is satisfied ${ }^{\mathrm{F}}$ in $\mathrm{OBJ}$ by some possible valuation $A^{\mathrm{d}}$ which is different from $A^{\mathrm{u}}$ at most at the $\mathrm{k}^{\mathrm{th}}$ place.

THEOREM 6 Axiom Iis valid ${ }^{\mathrm{F}}$.

PROOF: Implication to the right: Assume that $a \varepsilon b$ is $S A T_{O B J}^{F, u}$. Since, from the semantic interpretation of $\varepsilon$, a $\varepsilon b$ cannot be $S A T_{O B J}^{F, u}$, we, by $p \wedge \neg p \rightarrow q$, obtain the right side of the equivalence.

Implication to the left: Assume that the conjunction on the right side is $S A T_{O B J}^{F, u}$. Hence, $\exists_{c} c \varepsilon a$ is $S A T_{O B}^{F, u}$. Therefore, there exists the valuation $A^{s}$ that differs from $A^{\mathfrak{u}}$ at most in the assignment for $c$, such that $c \varepsilon a$ is $S A T_{O B}^{F, s}$. Since it cannot occur, according to our interpretation of $\varepsilon$, by $p \wedge \neg p \rightarrow q$ we obtain the left side of the equivalence. The reasoning is repeatable for any model and any valuation. Q.E.D.

THeOrem 7 Axiom 2 is valid ${ }^{\mathrm{F}}$. 
PROOF: Implication to the right: Assume $a \varepsilon b$ is $S A T_{O B J}^{F, u}$. But a $a b$ cannot be $S A T_{O B J}^{F, u}$. Hence, the right side of the equivalence in the axiom also is $\mathrm{SAT}_{\mathrm{O} \mathrm{BJ}}^{\mathrm{F}, \mathcal{u}}$ (since - in classical logic at least - contradiction implies any proposition).

Implication to the left: Assume $\exists_{c}(c \varepsilon a \wedge c \varepsilon b)$ is $S A T_{O B J}^{F, u}$. Therefore, c $c a \wedge$ $c \varepsilon b$ is satisfied ${ }^{F}$ in $O B J$ by a valuation $A^{s}$ which differs from $A^{u}$ at most on one place, namely in $V^{s}(c)$. Therefore, $c \varepsilon a$ is $S A T_{O B J}^{F, u}$. But it cannot be satisfied. From this contradiction, the left side of the equivalence results. Q.E.D.

THEOREM 8 Axiom 3 is valid ${ }^{\mathrm{F}}$.

The proof is exactly the same as in the case of Theorem 7

theorem 9 Axiom 4 is valid .

PROOF: The proof of implication to the right is the same, as in the proof of Theorem 7

Implication to the left: Assume that $\exists_{\mathfrak{c}}(a \varepsilon c \wedge c \varepsilon b)$ is $\operatorname{SAT}_{O B \mathrm{BJ}}^{\mathrm{F}, \mathrm{u}}$. Obviously, there is a valuation $A^{s}$ which differs from $A^{u}$ only in the value assigned co $c$, such that a $\varepsilon c \wedge c \varepsilon b$ is $S A T_{O B}^{F, s}$. Hence, a $a c$ is $S A T_{O B}^{F, s}$. But it cannot be the case. Therefore, from contradiction, we obtain the left side of the equivalence. Q.E.D.

\section{$3 \cdot 3$ NON-STANDARD INTERPRETATIONS OF $\subset$-AXIOMS}

\subsection{AXIOM 5}

In this axiom the primitive term is $\bar{\varepsilon}$-the functor of singular exclusion. In standard interpretation, intuitively, it yields true statement with its arguments $a, b$ iff $a$ is an unshared name, and $b$ does not name the object named by a (it does not matter, whether $b$ is unshared, shared, or empty).

Formally, we can accommodate the definition of satisfaction ${ }^{\varepsilon}$ to make $\bar{\varepsilon}$ the primitive term. Instead of first condition of the definition of satisfaction ${ }^{\varepsilon}$, we simply introduce:

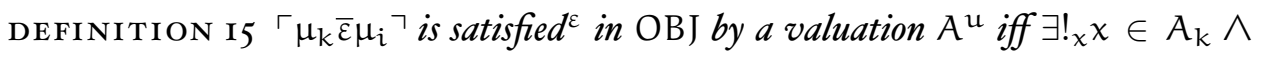
$\neg \exists_{y}\left[y \in A_{k} \wedge y \in A_{i}\right]$

We will now show that the Axiom 5 is valid also in some unintended interpretations of $\bar{\varepsilon}$.

According to the Definition 8 , $a \bar{\varepsilon} b$ is (in systems with $\varepsilon$ as primitive functor) defined by means of $a \varepsilon a \wedge \neg(a \varepsilon b)$. Let us follow this intuition, using the notion of satisfaction ${ }^{\Phi}$. We obtain: 
DEFINITION I6 $\mu_{\mathrm{k}} \bar{\varepsilon} \mu_{\mathrm{i}}$ is satisfied ${ }^{\Phi}$ in OBJ by $A^{u}$ iff $\mu_{\mathrm{k}} \varepsilon \mu_{\mathrm{k}} \wedge \neg\left(\mu_{\mathrm{k}} \varepsilon \mu_{\mathrm{i}}\right)$ is satisfied $^{\Phi}$ in $\mathrm{OBJ}$ by $\mathrm{A}^{\mathrm{u}}$.

In terms of model theory, it is equivalent to:

DEFINITION $\mathrm{I} 7 \mu_{\mathrm{k}} \bar{\varepsilon} \mu_{\mathrm{i}}$ is satisfied ${ }^{\Phi}$ in OBJ by $A^{\mathrm{u}}$ iff $\mathrm{V}^{\mathrm{u}}\left(\mu_{\mathrm{k}}\right)=\Phi \wedge \mathrm{V}^{\mathrm{u}}\left(\mu_{\mathrm{i}}\right) \neq \Phi$.

In an intuitive manner: $\mu_{k}$ is empty and $\mu_{i}$ is not empty.

theorem io Axiom 5 is valid ${ }^{\Phi}$.

PROOF:Implication to the right: Assume $a \bar{\varepsilon} b$ is $\operatorname{SAT}_{\mathrm{OBJ}}^{\Phi}$. Then, $\mathrm{V}^{\mathfrak{u}}(\mathrm{a})=\Phi \wedge$ $\mathrm{V}^{\mathfrak{u}}(\mathrm{b}) \neq \Phi$.

There exists a valuation $A^{s}$ which differs from $A^{u}$ at most in the fact, that $\mathrm{V}^{\mathrm{s}}(\mathrm{c})=\Phi$, such that $c \bar{\varepsilon} b \wedge \neg(\mathrm{c} \bar{\varepsilon} \mathrm{a})$ is $\operatorname{SAT}_{\mathrm{OBJ}}^{\Phi} \mathrm{s}$. It is so, because $\mathrm{V}^{\mathrm{s}}(\mathrm{c})=\Phi, \mathrm{V}^{\mathrm{s}}(\mathrm{b}) \neq \Phi$, and it is not the case that both: $\mathrm{V}^{\mathrm{s}}(\mathrm{c})=\Phi$ and $V^{s}(a) \neq \Phi$, I9

Hence, $\exists_{\mathrm{c}}(\mathrm{c} \bar{\varepsilon} \mathrm{b} \wedge \neg(\mathrm{c} \bar{\varepsilon} \mathrm{a}))$ is $\operatorname{SAT} \mathrm{T}_{\mathrm{OB} J \mathrm{~J}}^{\Phi}$.

Now, assume additionally that $c \bar{\varepsilon} \mathrm{d} \wedge d \bar{\varepsilon} e$ is $S A T_{\mathrm{OBJ}}^{\Phi}$. It means that $\Phi$, $\mathrm{V}^{\mathfrak{u}}(\mathrm{d}) \neq \Phi, \mathrm{V}^{\mathfrak{u}}(\mathrm{d})=\Phi$, and $\mathrm{V}^{\mathfrak{u}}(e) \neq \Phi$. Obviously, we have a contradiction. Hence, we obtain by $p \wedge \neg p \rightarrow q$ that semantic conditions of satisfaction $^{\Phi}$ of $c \bar{\varepsilon} a \vee d \bar{\varepsilon} a$ are fulfilled, and $c \bar{\varepsilon} a \vee d \bar{\varepsilon} a$ is $S A T_{O B J}^{\Phi, u}$.

Implication to the left: Assume $\exists_{\mathrm{c}}(\mathrm{c} \bar{\varepsilon} \mathrm{b} \wedge \neg(\mathrm{c} \bar{\varepsilon} \mathrm{a})) \wedge \forall_{\mathrm{c}, \mathrm{d}, e}(\mathrm{c} \bar{\varepsilon} \mathrm{d} \wedge \mathrm{d} \bar{\varepsilon} e \rightarrow \mathrm{d} \bar{\varepsilon} \mathrm{a})$ is $S_{A} T_{\mathrm{OBJ}}^{\Phi}$.

Since $\exists_{\mathfrak{c}}(c \bar{\varepsilon} b \wedge \neg(c \bar{\varepsilon} \mathfrak{a}))$ is $S A T_{O B J}^{\Phi, u}$, there exists a valuation $A^{s}$ differing from $A^{\mathfrak{u}}$ at most in the value assigned to $c$, such that $c \bar{\varepsilon} b \wedge \neg(c \bar{\varepsilon} \mathfrak{a})$ is $\mathrm{SAT}_{\mathrm{OBJ}}^{\Phi}$. It means that $\mathrm{V}^{\mathrm{s}}(\mathrm{c})=\Phi, \mathrm{V}^{\mathrm{s}}(\mathrm{b}) \neq \Phi$. Moreover, it implies that it is not the case, that both: $V^{s}(c)=\Phi$ and $V^{s}(a) \neq \Phi$. Since $\mathrm{V}^{\mathrm{s}}(\mathrm{c})=\Phi$, obviously $\mathrm{V}^{\mathrm{s}}(\mathrm{a})=\Phi$. According to our understanding of $A^{s}, V^{\mathfrak{u}}(a)=V^{s}(a)$ and $V^{\mathfrak{u}}(b)=V^{s}(b)$. Therefore, $V^{\mathfrak{u}}(b) \neq \Phi$ and $\mathrm{V}^{\mathfrak{u}}(\mathrm{a})=\Phi$. Therefore, $a \bar{\varepsilon} b$ is $\operatorname{SAT}_{\mathrm{OB}}^{\Phi}, \mathrm{s}$. This reasoning can be repeated for any OBJ and any $A^{u}$. This ends the proof. Q.E.D.

We can also follow Definition 8 using the notion of satisfaction ${ }^{\mathrm{F}}$. We obtain:

DEFINITION I8 $a \bar{\varepsilon} b$ is satisfied ${ }^{\mathrm{F}}$ in OBJ by $\mathrm{A}^{\mathfrak{u}}$ iff $^{\mathrm{u}}(\mathrm{a}) \neq \mathrm{V}^{\mathfrak{u}}(\mathrm{a})$.

Theorem in Axiom [ is valid $^{\mathrm{F}}$.

PROOF: Implication to the right: Assume $a \bar{\varepsilon} b$ is $S_{A} T_{O B J}^{F, u}$. Obviously, it cannot be satisfied. From this contradiction, we obtain the right side of the equivalence.

\footnotetext{
${ }^{19}$ Since $V^{u}(a)=V^{s}(a)$ and $V^{u}(b)=V^{s}(b)$.
} 
Implication to the left: Assume $\exists_{\mathrm{c}}(\mathrm{c} \bar{\varepsilon} \mathrm{b} \wedge \neg(\mathrm{c} \bar{\varepsilon} \mathrm{a})) \wedge \forall_{\mathrm{c}, \mathrm{d}, \mathrm{e}}(\mathrm{c} \bar{\varepsilon} \mathrm{d} \wedge \mathrm{d} \bar{\varepsilon} e \rightarrow \mathrm{d} \bar{\varepsilon} \mathrm{a})$ is $S A T_{O B J}^{\mathrm{F}, u}$.

Since $\exists_{\mathfrak{c}}(c \bar{\varepsilon} b \wedge \neg(c \bar{\varepsilon} a))$ is $S A T_{O B J}^{F, u}$, there exists a valuation $A^{s}$ differing from $A^{u}$ at most in the value assigned to $c$, such that $c \bar{\varepsilon} b \wedge \neg(c \bar{\varepsilon} a)$ is $S A T_{O B J}^{F, s}$. It implies that $c \bar{\varepsilon} b$ is $S A T_{O, B}^{F}$. But it cannot be satisfied. Hence, by contradiction, we obtain the left side of the equivalence. Q.E.D.

\section{$3.3 .2 \quad$ АХIOM 6}

Sobociński's axiomatization has $<$ as the only primitive functor. Let us follow the Definition 9 according to the definition of satisfaction ${ }^{\Phi}$. Our semantics yields the result that interpretation ${ }^{\Phi}$ of $<$ is the same as the interpretation ${ }^{\Phi}$ of $\varepsilon$.

Theorem i2 Axiom 6 is valid ${ }^{\Phi}$.

PROOF:Implication to the right: Assume $\mathrm{a}<\mathrm{b}$ is $\operatorname{SAT}_{\mathrm{OBJ}}^{\Phi} \mathrm{u}$. Hence $\mathrm{V}^{\mathrm{u}}(\mathrm{a})=$ $V^{\mathfrak{u}}(b)=\Phi$. There exists a valuation $A^{s}$ differing from $A^{\mathfrak{u}}$ at most in the value assigned to $c$, such that $V^{s}(c)=\Phi$. Obviously $c<a$ is $S A T_{O B J}^{\Phi, s}$. Therefore, $\exists_{\mathrm{c}} \mathrm{c}<\mathrm{a}$ is $\mathrm{SAT}_{\mathrm{OBJ}}^{\Phi, \mathrm{u}}$.

Now assume additionally, that $c<a$ is $S A T_{O B J}^{\Phi}$. . Then $V^{\mathfrak{u}}(c)=\Phi$. Since $\mathrm{V}^{\mathfrak{u}}(\mathrm{b})=\Phi$, it is clear that $\mathrm{V}^{\mathrm{u}}(\mathrm{c})=\mathrm{V}^{\mathfrak{u}}(\mathrm{b})=\Phi$, and $\mathrm{c}<\mathrm{b}$ is $S A T_{O B}^{\Phi}, \mathfrak{u}$.

Therefore $\forall_{c}(c<a \rightarrow c<b)$ is $\operatorname{SAT}_{\mathrm{OBJ}}^{\Phi}$. Then also $\forall_{\mathrm{c}}\left(\mathrm{c}<\mathrm{a} \wedge \forall_{\mathrm{d}}(\mathrm{d}<\right.$ $\mathrm{c} \rightarrow \mathrm{c}<\mathrm{d}) \rightarrow \mathrm{c}<\mathrm{b})$ is $\operatorname{SAT}_{\mathrm{OB} J}^{\Phi}$.

Implication to the left: Assume $\exists_{\mathrm{c}} \mathrm{c}<\mathrm{a} \wedge \forall_{\mathrm{c}}\left(\mathrm{c}<\mathrm{a} \wedge \forall_{\mathrm{d}}(\mathrm{d}<\mathrm{c} \rightarrow \mathrm{c}<\mathrm{d}) \rightarrow\right.$ $\mathrm{c}<\mathrm{b})$ is $\mathrm{SAT}_{\mathrm{OBJ}}^{\Phi}$.

Since $\exists_{c} c<a$ is $\operatorname{SAT}_{O B J}^{\Phi}, u$, there exists a valuation $A^{s}$ differing from $A^{u}$ at most in the value assigned to $c$, such that $V^{s}(c)=V^{s}(a)=\Phi$. Since $\mathrm{V}^{\mathrm{s}}(\mathrm{a})=\mathrm{V}^{\mathfrak{u}}(\mathrm{a})$, clearly $\mathrm{V}^{\mathfrak{u}}(\mathrm{a})=\Phi$.

According to our assumption, $\forall_{\mathrm{c}}\left(\mathrm{c}<\mathrm{a} \wedge \forall_{\mathrm{d}}(\mathrm{d}<\mathrm{c} \rightarrow \mathrm{c}<\mathrm{d}) \rightarrow \mathrm{c}<\mathrm{b}\right)$ is SAT O $_{\mathrm{B} J}^{\mathrm{u}}$. Moreover, $\forall_{\mathrm{d}}(\mathrm{d}<\mathrm{c} \rightarrow \mathrm{c}<\mathrm{d})$ is valid ${ }^{\Phi}$, since identity for sets is symmetrical. Hence $\forall_{c}(c<a \rightarrow c<b)$ is $\operatorname{SAT}_{O B J}^{\Phi, u}$.

Obviously $a<a$ is $\operatorname{SAT}_{O B J}^{\Phi, \mathfrak{u}}\left(\right.$ because $\left.\mathrm{V}^{\mathfrak{u}}(\mathrm{a})=\mathrm{V}^{\mathfrak{u}}(\mathrm{a})=\Phi\right)$. Then, in accordance with the valid implication above $\left(\forall_{c}(c<a \rightarrow c<b)\right.$ ), also $a<b$ is $S_{A T}^{\Phi} T_{O B J}^{\Phi}$. This result ends our proof. Q.E.D.

We can also follow this definition by means of the definition of satisfaction ${ }^{\mathrm{F}}$. The resulting interpretation ${ }^{\mathrm{F}}$ of $<$ is the same as the interpretation ${ }^{\mathrm{F}}$ of $\varepsilon$.

theorem i3 Axiom 6 is valid ${ }^{\mathrm{F}}$. 
PROOF: Implication to the right: Assume $a<b$ is $S_{A T}^{F, u}$. Since it cannot be satisfied, we have contradiction, and the right side of the equivalence follows obviously.

Implication to the left: Assume $\exists_{c} c<a$ is $\operatorname{SAT}_{O B \mathrm{BJ}}^{\mathrm{F}, \mathrm{u}}$. Then for some valuation $A^{s}$ differing at most in the value assigned to $c, c<a$ is $S A T_{O B}^{F, s}$. Since it cannot be the case, we obtain contradiction, which yields the needed statement. Q.E.D.

\section{$3 \cdot 3 \cdot 3 \quad$ Aхгом 7}

The primitive term of Ontology based on this axiom is $\subset$. Let us find a nonstandard interpretation of this functor, following the definition of satisfaction ${ }^{\Phi}$ and the Definition II It is defined by means of $\forall_{c}(c \varepsilon a \rightarrow c \varepsilon b)$. Hence, we can introduce the following:

DEFINITION I9 $\left\ulcorner\mu_{\mathrm{k}} \subset \mu_{\mathrm{i}}\right\urcorner$ is satisfied ${ }^{\Phi}$ in $\mathrm{OBJ}$ by a valuation $\mathrm{A}^{\mathrm{u}}$ if and only if

$$
A_{k}=\Phi \rightarrow A_{i}=\Phi
$$

It is clear that this interpretation is far from standard. We shall now prove the following:

Theorem i4 Axiom 7 is valid ${ }^{\Phi}$.

PROOF: Implication to the right: Assume $a \subset b$ is $S A T_{O B}^{\Phi, u}$. It implies that if $\mathrm{V}^{\mathfrak{u}}(\mathrm{a})=\Phi$ then also $\mathrm{V}^{\mathfrak{u}}(\mathrm{b})=\Phi$.

Assume additionally that $\mathrm{c} \subset \mathrm{a}$ is $\operatorname{SAT}_{\mathrm{OBJ}}^{\Phi, \mathrm{u}}$ and $\mathrm{V}^{\mathrm{u}}(\mathrm{c})=\Phi$. It means, that if $\mathrm{V}^{\mathfrak{u}}(\mathrm{c})=\Phi$, then also $\mathrm{V}^{\mathfrak{u}}(\mathrm{a})=\Phi$. From this assumptions we obtain that $\mathrm{V}^{\mathfrak{u}}(\mathrm{a})=\Phi$, and, since $\mathrm{V}^{\mathfrak{u}}(\mathrm{a})=\Phi \rightarrow \mathrm{V}^{\mathfrak{u}}(\mathrm{b})=\Phi$, obviously $\mathrm{V}^{\mathfrak{u}}(\mathrm{b})=\Phi$.

Therefore, also $c \subset b$ is $\operatorname{SAT}_{\mathrm{OBJ}}^{\Phi} \mathrm{u}$.

Implication to the left: This part of the proof will be indirect. Assume the following:

I. $a \subset b$ is not $S A T_{\mathrm{OB} \mathrm{J}}^{\Phi}$.

2. $\forall_{c}\left(c \subset a \wedge \forall_{d, e}(d \subset c \rightarrow c \subset d \vee d \subset e) \rightarrow c \subset b\right)$ is $S_{A} T_{O B J}^{\Phi, ~}$.

Obviously (by the semantic correlate of universal instantiation) also $\left.a \subset a \wedge \forall_{d, e}(d \subset a \rightarrow a \subset d \vee d \subset e) \rightarrow a \subset b\right)$ is $\operatorname{SAT}_{O B J}^{\Phi, u}$.

It is clear that $V^{\mathfrak{u}}(a)=\Phi \rightarrow V^{\mathfrak{u}}(a)=\Phi$. Hence, $a \subset a$ is $\operatorname{SAT}_{O B J}^{\Phi}, \mathfrak{u}$.

Now, from the first assumption, it is not the case that $\mathrm{V}^{\mathrm{u}}(\mathrm{a})=\Phi \rightarrow$ $\mathrm{V}^{\mathfrak{u}}(\mathrm{b})=\Phi$. Hence $\mathrm{V}^{\mathfrak{u}}(\mathrm{a})=\Phi \wedge \mathrm{V}^{\mathfrak{u}}(\mathrm{b}) \neq \Phi$ is $S A T_{\mathrm{OBJ}}^{\Phi}$. 
Now assume that $d \subset a$ is $S A T_{O B J}^{\Phi, u}$. Clearly, no matter what the value of $\mathrm{d}$ is, either $\mathrm{V}^{\mathfrak{u}}(\mathrm{d})=\Phi$, or $\mathrm{V}^{\mathfrak{u}}(\mathrm{d}) \neq \Phi$. In the first case, $\mathrm{V}^{\mathrm{u}}(\mathrm{a})=$ $\Phi \rightarrow \mathrm{V}^{\mathfrak{u}}(\mathrm{d})=\Phi$, and $\mathrm{a} \subset \mathrm{d}$ is $\operatorname{SAT}_{\mathrm{OBJ}}^{\Phi, \mathrm{u}}$. In the second case, obviously $\mathrm{V}^{\mathfrak{u}}(\mathrm{d})=\Phi \rightarrow \mathrm{V}^{\mathfrak{u}}(e)=\Phi$ (no matter, what the value of $e$ is, since the antecedent is false). In both cases, $a \subset d \vee d \subset e$ is $S_{A T}$, $T^{\prime}$.

Therefore, $\forall_{d, e}(d \subset a \rightarrow a \subset d \vee d \subset e)$ is $\operatorname{SAT}_{O B}^{\Phi} \mathrm{u}^{\mathrm{u}}$.

Now, by modus ponendo ponens, we obtain the consequence that $\mathrm{a} \subset \mathrm{b}$ is $S A T_{\mathrm{OBJ}}^{\Phi, \mathrm{u}}$. This gives us contradiction with the assumption of indirect proof. This result ends our proof. Q.E.D.

Clearly, our method works also when we follow the definition of satisfaction ${ }^{\mathrm{F}}$. According to Definition II we obtain:

DEFINITION $20\left\ulcorner\mu_{\mathrm{k}} \subset \mu_{\mathrm{i}}\right\urcorner$ is satisfied ${ }^{\mathrm{F}}$ in $\mathrm{OBJ}$ by a valuation $\mathrm{A}^{\mathrm{u}}$ if and only if $A_{k}=A_{k}$.

In this interpretation $\subset$ is a verum functor which yields the value I for any arguments. We claim:

TheOrem 5 Axiom 7 is valid ${ }^{\mathrm{F}}$.

PROOF: Implication to the right: Assume $\mathrm{a} \subset \mathrm{b}$ is $\mathrm{SAT}_{\mathrm{OB}}^{\mathrm{F}, \mathrm{u}}$.

Clearly, $c \subset b$ is $S A T_{O B J}^{F, u}$, since $V^{u}(c)=V^{\mathfrak{u}}(c)$. Therefore, also the whole implication on the right side of equivalence is $S A T_{\mathrm{O}, \mathrm{BJ}}^{\mathrm{F} J}$.

Implication to the left: Similarly $-a \subset b$ is $\operatorname{SAT}_{O B J}^{F, u}\left(\right.$ because $V^{u}(a)=$ $\mathrm{V}^{\mathrm{u}}(\mathrm{a})$ ). Therefore whole the implication which has $\mathrm{a} \subset \mathrm{b}$ as consequent is $S A T_{O B J}^{F, u}$. This ends our proof. Q.E.D.

\subsection{SOME OTHER NON-STANDARD INTERPRETATIONS}

As we have said elsewhere [2I], there are $2^{16}$ possible interpretations of a given functor of the category $\frac{s}{n, n}$. The claim that an axiom determines exactly one of this meanings is therefore quite a strong claim. In this section we shall indicate that the two interpretations presented above are not the only two possible non-standard interpretation.

However, our treatment of this issue will be less precise that the deliberations led to this point. We will only prove our theorems for the Axiom 4 Clearly, those claims can be applied to other axioms. We omit these theorems and their proofs just for the sake of convenience. We can, for example, introduce the following inductive condition of satisfaction.:

DEFINITION 2I $\mu_{k} \varepsilon \mu_{i}$ is satisfied ${ }^{\alpha}$ in OBJby $A^{u}$ iff $\exists_{x \neq y}\left[x \in A_{k} \wedge y \in A_{k} \wedge A_{k}=\right.$ $\left.A_{i}\right]$. 
In other words, in interpretation ${ }^{\alpha}$ we understand $\varepsilon$ as identity of extensions having more that one element.

theorem i6 Axiom 4 is valid ${ }^{\alpha}$.

PROOF: Implication to the right: Assume $a \varepsilon b$ is $\operatorname{SAT}_{O B J}^{\alpha, u}$. It means that $\mathrm{V}^{\mathrm{u}}(\mathrm{a})$ and $\mathrm{V}^{\mathrm{u}}(\mathrm{b})$ are non empty, identical and not unary. There is a valuation $A^{s}$ which differs from $A^{u}$ at most in the fact that $V s(c)=V^{s}(a)$. Clearly $a \varepsilon c \wedge c \varepsilon b$ is $S A T_{O B J}^{\alpha, s}$.

Therefore, $\exists_{\mathrm{c}}(a \varepsilon c \wedge c \varepsilon b)$ is $\mathrm{SAT}_{\mathrm{OBJ}}^{\alpha, u}$.

Implication to the left: Assume that $\exists_{\mathfrak{c}}(a \varepsilon c \wedge c \varepsilon b)$ is $S A T_{O B J}^{\alpha, u}$. Then, there is a valuation $A^{s}$ which differs from $A^{u}$ at most in the fact that $V s(c)=$ $V^{s}(a)$. Clearly a $a c \wedge c \varepsilon b$ is $\operatorname{SAT}_{O B J}^{\alpha, s}$ and sets $V^{s}(a), V^{s}(b), V^{s}(c)$ are identical, nonempty and not unary.

Since $V^{s}(a)=V^{\mathfrak{u}}(a)$ and $V^{s}(b)=V^{\mathfrak{u}}(b)$, it is obvious that $V^{\mathfrak{u}}(a)$ and $\mathrm{V}^{\mathfrak{u}}(\mathrm{b})$ are identical, nonempty and not unary. Therefore, $a \varepsilon b$ is $S A T_{O B J}^{\alpha, u}$. This ends the proof. Q.E.D.

DEFINITION $22 \mu_{k} \varepsilon \mu_{i}$ is satisfied ${ }^{\beta}$ in OBJ by $A^{u}$ iff $\exists !_{x}\left[x \in A_{k} \wedge A_{k}=A_{i}\right]$.

According to the definition of satisfaction ${ }^{\beta}$, we interpret $\varepsilon$ as the identity of unary extensions of given arguments.

TheOrem 7 Axiom 4 is valid ${ }^{\beta}$.

The proof of Theorem 17 is very similar to the proof of Theorem 16

DEFINITION $23 \mu_{k} \varepsilon \mu_{i}$ is satisfied ${ }^{\gamma}$ in OBJ by $A^{u}$ iff $\exists_{x}\left[x \in A_{k} \wedge A_{k}=A_{i}\right]$.

According to satisfaction ${ }^{\gamma}$, we interpret $\varepsilon$ as identity of nonempty extensions of given arguments.

THeOrem is Axiom 4 is valid ${ }^{\gamma}$.

As before, the proof of Theorem $\mathrm{I} 8$ is very similar to the proof of Theorem $\mathrm{I} 6$

The list of hitherto given interpretations of primitive functors of Leśniewski's Ontology is not a complete list of possible interpretations of primitive constants of Ontology which keep axioms valid.

\subsection{A GENERAL CASE - NUMERICAL IDENTITY}

Some of the interpretations of $\varepsilon$ mentioned above represent a particular instances of a more general situation. These are, to speak freely, these interpretations of $\varepsilon$, which imply identity ${ }^{20}$

${ }^{20}$ Strictly speaking: a given interpretation $\psi$ of atomic sentences defined by the condition of the kind: ' $a \varepsilon b$ is $S A T_{O B J}^{\psi, u}$ iff $\psi(a, b)$ ' implies identity iff $\psi(a, b)$ implies $V^{u}(a)=V^{u}(b)$. 
In a language with identity of individuals we can easily define predicates: 'has exactly (at least, at most) $n$ elements'. Hence, we can introduce $\mathbf{x}_{0}$ definitions of satisfaction by constructing the basic condition according to this scheme:

[IDENTITY SCHEME] $\mu_{k} \varepsilon \mu_{i}$ is satisfied ${ }^{n(\geqslant n / \leqslant n)}$ in OBJ by $A^{u}$ iff $A_{k}=A_{i}$ and $A_{k}$ has exactly (at least/ at most) $n$ elements.

where $\mathrm{n}$ is a natural number. We observe:

THEOREM I9 Any satisfaction defined in accordance with the IDENTITY SCHEME makes all axioms given in this paper valid.

We have already given proofs of this theorem for some particular notions of numerical satisfaction - satisfaction ${ }^{\Phi}$, satisfaction ${ }^{\alpha}$, satisfaction ${ }^{\beta}$, satisfaction ${ }^{\gamma}$. Proof of this theorem is an easy generalization of proofs of corresponding claims for these interpretations. Such proofs have been already given. The proof needed now is constructed from those by replacing a particular numerical description ${ }^{27}$ by 'has (have) exactly (at least/ at most) $n$ elements'.

\section{RULE OF EXTENSIONALITY}

We shall begin with the application of this rule to the basic language, i. e. with the schema

$$
\text { From } \forall_{x}[x \varepsilon \lambda \equiv \chi \varepsilon \delta] \text { infer } \forall_{\chi}[\chi(\lambda) \equiv \chi(\delta)]
$$

It will be convenient to begin with a lemma saying that if the rule works for $\chi=\varphi$ and for $\chi=\psi$, then it works for expressions composed from $\varphi$ and $\psi$ by the rules of $\mathrm{BL}$ (conjunction, negation, quantification). This is the most trivial part of a full proof of our main claim for this rule. We can prove it in general, and later on prove only the first condition of inductive proof for different cases separately.

LeMma I If the two schemata are validity-preserving:

$$
\begin{aligned}
& \text { From } \forall_{x}\left[x_{\varepsilon} \lambda \equiv \chi_{\varepsilon} \delta\right] \text { infer }[\varphi(\lambda) \equiv \varphi(\delta)] \\
& \text { From } \forall_{x}\left[x_{\varepsilon} \lambda \equiv x_{\varepsilon} \delta\right] \text { infer }[\psi(\lambda) \equiv \psi(\delta)]
\end{aligned}
$$

then, also the following schemata are validity-preserving:

I. From $\forall_{x}[x \varepsilon \lambda \equiv x \varepsilon \delta]$ infer $[\varphi(\lambda) \wedge \psi(\lambda) \equiv \varphi(\delta) \wedge \psi(\delta)]$

2. From $\forall_{x}[x \varepsilon \lambda \equiv x \varepsilon \delta]$ infer $[\neg \varphi(\lambda) \equiv \neg \varphi(\delta)]$

3. From $\forall_{x}\left[x_{\varepsilon} \lambda \equiv x_{\varepsilon} \delta\right] \operatorname{infer}\left[\forall_{\mu} \varphi(\lambda) \equiv \forall_{\mu} \varphi(\delta)\right]$

\footnotetext{
${ }^{21}$ Like, for example, 'is non-empty' or 'has at least two elements'.
} 
PROOF: Assume that the rule is validity-preserving for $\varphi$ and $\psi$. Assume that $\forall_{x}\left[x_{\varepsilon} \lambda \equiv x_{\varepsilon} \delta\right]$ is valid. Hence, we can infer $\varphi(\lambda) \equiv \varphi(\delta)$ and $\psi(\lambda) \equiv \psi(\delta)$. The cases of conjunction and negation result from, respectively:

$$
\begin{gathered}
(p \equiv q) \wedge(r \equiv s) \rightarrow((p \wedge r) \equiv(q \wedge s)) \\
(p \equiv q) \rightarrow(\neg p \equiv \neg q)
\end{gathered}
$$

Regarding quantification: Since $\forall_{\mu}(\varphi(\lambda))$ and $\forall_{\mu}(\varphi(\delta))$ must have $\lambda$ and $\delta$ as free variables ${ }^{22}$, clearly $\lambda \neq \mu$ and $\delta \neq \mu$. We have now two possibilities: either $\mu$ occurs in $\varphi$, or not. If it does not occur in $\varphi$, it, to say it loosely, it does not contribute to the value of $\varphi(\lambda)$ or $\varphi(\delta)$. If $\mu$ occurs in $\phi$ as a bound variable, the addition of superfluous quantification also changes nothing in the value of whole expressions. So the only interesting case remaining is that $\mu$ occurs in $\phi$ as a free variable. (Note that we already have $\varphi(\lambda) \equiv \varphi(\delta)$.)

If this is valid, then for any $O B J$ and for any valuation of free variables ( $\mu$ among them) $\varphi(\lambda)$ is equivalent to $\varphi(\delta)$. Clearly, then, [for any valuation of $\mu, \varphi(\lambda)$ ] is equivalent to [for any valuation of $\mu, \varphi(\delta)$ ]. Hence, $\left[\forall_{\mu} \varphi(\lambda) \equiv \forall_{\mu} \varphi(\delta)\right]$ is valid. Q.E.D.

With the aid of this result, we can easily give proofs that the rule of extensionality is validity-preserving for a given interpretation. Since LemmaI works for any interpretation, to complete an inductive proof it will suffice to prove a claim about the basic condition only.

THEOREM 20 Rule of extensionality is validity ${ }^{\Phi}$-preserving.

PROOF: To prove this claim we shall prove that if $\forall_{x}\left[x \varepsilon \lambda \equiv x_{\varepsilon} \delta\right]$ is valid ${ }^{\Phi}$, then the following schemata are valid ${ }^{\Phi}$ :

I. $\gamma \varepsilon \lambda \equiv \gamma \varepsilon \delta$

2. $\lambda \varepsilon \gamma \equiv \delta \varepsilon \gamma$

In fact, the first case is obvious (check our semantics for the universal quantifier). The second results from the fact that the relation of 'being simultaneously empty' is symmetrical.

Indeed, these two cases exhaust all possibilities of 'atomic' formulas in which both $\lambda$ and $\delta$ occur at the same position. Together with Lemma I this fact yields a complete inductive proof for $\mathrm{BL}$.

Q.E.D.

${ }^{22}$ See the formulation of the rule of extensionality. 
We can prove a more general claim:

THEOREM 2 I Rule of extensionality preserves validity in $\mathrm{BL}$ for any interpretation of $\varepsilon$ constructed in accordance with the schema of numerical identity.

Proof: Assume that

$$
\forall x(x \varepsilon \lambda \equiv x \varepsilon \delta)
$$

is valid. It follows that for any OBJ and any valuation $u$ of name variables in this model, any (and only) subset of OBJ which is $\mathrm{nI}$ with $\mathrm{EV}^{\mathrm{u}}(\lambda)$ is $\mathrm{nI}$ with $\mathrm{EV}^{\mathfrak{u}}(\delta){ }^{23}$ This implies that $\mathrm{EV}^{\mathfrak{u}}(\lambda)$ and $\mathrm{EV}^{\mathfrak{u}}(\delta)$ are $\mathrm{nI}$ with each other (and each of them is $\mathrm{nI}$ with itself). If these semantic values are identical, it does not matter which of these: $\lambda$ or $\delta$, occur in an expression - since satisfaction is defined in terms of extensions of names and name variables and their power (the latter is preserved by identity). So, in fact, always any expression with $\lambda$ is equivalent with the same expression after replacing $\lambda$ by $\delta$. Q.E.D.

This proof seems to be quite plausible, mutatis mutandis, for the case of nameand sentence-formative functors. Hence:

HY РОTHESIS 3 Rule of extensionality preserves validity in the full language of Ontology for any interpretation of $\varepsilon$ constructed in accordance with the schema of numerical identity.

Almost all interpretations considered in this paper fall under the schema for numerical identity. The only interpretation which need an independent proof is the notion of satisfaction ${ }^{\mathrm{F}}$.

THEOREM 22 Rule of extensionality preserves validity ${ }^{\mathrm{F}}$ in $\mathrm{BL}$.

PROOF: We need to prove only the basic case (for atomic expressions). Indeed, the antecedent of the rule of extensionality itself is a valid expression, for any $\delta$ and $\lambda$ (both arguments of the equivalence are always false) ${ }^{24}$ Now, clearly all atomic sentences are equivalent - they are always false. Thanks to Lemma I we obtain a full inductive proof. Q.E.D.

Presumably, the above proof works, mutatis mutandis, for the full language of Ontology.

${ }^{23}$ We say shortly ' $n I$ ' instead of 'n-numerically identical' where 'n-numerical identity' replaces 'being identical and having exactly/at least/at most $n$ elements'. We hope that this abbreviation will not cause any ambiguity.

${ }^{24}$ It does not indicate, however, that we can (syntactically) prove this antecedent in the axiomatic system under consideration. 


\section{RULE OF DEFINITION}

It is quite clear that:

THEOREM 23 The rule of definition is validity ${ }^{\mathrm{F}}$-preserving in the basic language of Ontology.

PROOF: For the case of definitions proposition-formative functors, the case is simple, because the interpretation of $\varepsilon$ is not relevant for the validity of such definitions. For the case of definitions of nominal constants and of name-formative functors note, that such a definition:

(a) Has an atomic expression as definiendum.

(b) Has at least one atomic expression as a part of a conjunction in the definiens.

Since any atomic expression is unsatisfiable ${ }^{\mathrm{F}}$, it follows that both the definiendum and definiens of any such definition are unsatisfiable. Hence, any such definition (since it is an equivalence) is valid. Q.E.D.

Nevertheless, an anonymous referee of this paper has given an interesting argument for the claim that in the interpretation ${ }^{\Phi}$ of $\varepsilon$ the rule of definition 'is not truth preserving' in the $\Phi$-interpretation. This arguments sheds some light on important issues connected with this rule. I am really grateful to this referee, because his comment, relevant indeed, reminded me of some issues neglected in the first version of this paper.

Let us start with the argument itself before adding a comment. ${ }^{25}$

ARGUMENT

The definition of the empty name is:

$$
\mathrm{D} \bigcap: \quad \forall_{\mathrm{a}}(\mathrm{a} \varepsilon \bigcap \equiv \mathrm{a} \varepsilon \mathrm{a} \wedge \neg \mathrm{a} \varepsilon \mathrm{a})
$$

When we introduce this definition, we can prove $\neg \bigcap \varepsilon \bigcap$ in Ontology, which is false according to $\Phi$-interpretation.

comment In fact, the rule of definition allows us to introduce $\mathrm{D} \cap$ as a theorem. Since it is only 'one-step'-rule, its validity-preserving property consists in the fact that any expression introduced by means of this rule is valid. ${ }^{26}$

\footnotetext{
${ }^{25}$ I wholeheartedly hope that the referee does not mind quoting his argument.

${ }^{26}$ Please note, that for our purpose it suffices to consider the property of being validitypreserving (which in fact, for rules of introducing sentences, coincides with being truthpreserving). As we will see, expressions introduced by means of this rule will not have to be sentences. Especially, the so-called quasi-constants (we shall explain this notion soon) are, in some sense, free in such 'definitions' in some non-standard interpretations. To resume - validity preserving is the weakest property we need, so we will be concerned with it (and not with truth-preserving property) in our further deliberations.
} 
On the other hand, it is a wholly different question, whether expressions introduced by means of this rule determine constant denotations of expressions defined in these definitions. This may be clearly seen on the example of $\mathrm{D} \cap$. Indeed, we can see that it does not 'determine' the standard denotation of $\bigcap$. Colloquially speaking, we may read $\mathrm{D} \cap$ in our $\Phi$-interpretation rather as ${ }^{27}$

For any name $a, a$ is empty together with $\bigcap$ iff ( $a$ is empty with itself and it is not the case that $a$ is empty with itself).

It follows that for no $a$ it is the case that $a \varepsilon \bigcap$. But, in our interpretation $(\Phi)$ it does not mean that $\bigcap$ is the empty name. To the contrary, it means that no name is empty together with $\bigcap$, so we should rather call $\cap$ 'the non-empty name' (whether the usage of 'the' before 'non-empty name' is legitimate is itself debatable).

This fact indicates that when we apply the $\Phi$-interpretation (and possibly, some other interpretations) to definitions of Ontology, they may not determine the semantics of defined expressions. ${ }^{28}$ We can now understand $\bigcap$ in many different ways, as far as we assign in any OBJ a non-empty set as its semantic correlate.

Regardless, if we interpret $\bigcap$ as a non-empty name (as we are forced to do by $\mathrm{D} \cap$ in the $\Phi$-interpretation), no surprise (and, moreover, no contradiction) that we obtain as a result:

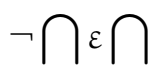

But we cannot read it as 'the empty name is not empty together with itself' (which is false), but rather: 'a non-empty name is not empty together with itself' (which is true).

To summarize, when we change the meaning of constants of a given language, this shapes also our account of definitions built in this language - we have to apply our new semantics also to definitions.

GENERAL CONSIDERATIONS Nevertheless, the referee is quite right: the rule is not validity-preserving in some non-standard interpretations. It surely is not validity-preserving in any numerical interpretation where $n \geqslant 2$, if we put no restrictions on the validity/unsatisfiability of $\chi$ occurring in the definition. Consider the following:

$$
\mathrm{D} * \quad \forall_{\mathrm{a}}[\mathrm{a} \varepsilon * \equiv \mathrm{a} \varepsilon \mathrm{a} \wedge(\mathrm{a} \varepsilon \mathrm{a} \vee \neg \mathrm{a} \varepsilon \mathrm{a})]
$$

This expression obviously fails in numerous cases of n-identity. For example, read $\varepsilon$ as denoting the relation: 'has at most 5 elements and is identical

\footnotetext{
${ }^{27}$ I hope that this reading will suffice. Anyone who feels that the full formal reading is necessary, is free to read it accordingly.

${ }^{28} \mathrm{It}$ is interesting question, whether definitions originally in fact determined such semantics. Anyway, even if they do not determine this semantics, it does not mean that they are not valid.
} 
with', and let $\chi$ be valid (like in $\mathrm{D} *$ ). Let $\mathrm{OBJ}$ be a model under consideration. We obtain the result that $*$ denotes a subset $V(*)$ of OBJ, such that any $A \subseteq O B J$ which has at most $n$ elements, (has at most $n$ elements and) is identical with $\mathrm{V}(*)$. Let $\mathrm{OBJ}=\{x, y, z\}, \mathrm{B}=\{1,2\}, \mathrm{C}=\{2,3\}, \mathrm{B}, \mathrm{C} \subseteq \mathrm{OBJ}$. Clearly both $B$ and $C$ have at most 5 elements. Hence, $A=V(*), B=V(*)$. Therefore: $A=B$, which is false.

There are, however, at least two non-standard interpretations, in which this rule is validity preserving - F-interpretation, and $\Phi$-interpretation.

Generally, the question whether the rule of definition is validity preserving in non-standard interpretations gives rise to quite complicated deliberations. The restrictions put on possible non-standard interpretation of $\varepsilon$ by introducing the rule of definition (or the other way round - the restrictions put on this rule by introducing some non-standard interpretations) constitute a wide problem which I cannot discuss fully in this paper. I hope that I will be able to come back to this issue in a separate paper.

\section{SUMMARY}

We have proposed quite an intuitive understanding of the expression 'an axiom determines the meaning of the only specific constant occurring in it' and 'the meaning of a given constant is determined axiomatically'. We have introduced some basic semantics for functors of category $\frac{s}{n, n}$ of Leśniewski's Ontology. Using this results we have proven that the popular claim that axioms of Ontology determine the meaning of primitive constants (functors), or that an axiomatic basis of Ontology determines the meaning of the primitive constant of this basis, is false 29

\section{REFERENCES}

[I] Ajdukiewicz, K. Sprawozdanie z działalności Seminarium Pracowni Logiki Polskiej Akademii Nauk za IV kwartat 1955 r., Studia Logica, V, 1957

[2] Borkowski, L., Wprowadzenie do Logiki i Teorii Mnogości, Towarzystwo Naukowe KU L, Lublin I99I

[3] Canty, J., T., Ontology: Lésnierwskis Logical Language, "Leśnierwskiłs Systems. Ontology and Mereology, Editors: Jan T. J. Srzednicki, V. F. Rickey, J. Czelakowski, Polish Academy of Sciences, Institute of Philosophy and Sociology, Matrinus Nijhoff Publishers, The Hague, I984, pp. I49-I63

\footnotetext{
${ }^{29}$ As far as we do not require completeness with respect to a given non-standard interpretation to be a necessary condition of 'being a theory of' the functor in this interpretation. In fact, our approach which omits this condition seems to be quite plausible - at least as far, as we claim that standard arithmetics, in some sense, is a theory of numbers and some operations on them: addition, etc. (though we seem to have some problems with the completeness of arithmetics).
} 
[4] Hiż, H., Descriptions in Russell's theory and in ontology, [in:] Studia Logica,1977, $\mathrm{xxxVI}, 4$, pp. $27 \mathrm{I}-283$

[5] Iwanuś, On Leśniereskiss Elementary Ontology, Lé́nierwskiłs Systems. Ontology and Mereology, Editors: Jan T. J. Srzednicki, V. F. Rickey, J. Czelakowski, Polish Academy of Sciences, Institute of Philosophy and Sociology, Matrinus Nijhoff Publishers, The Hague, 1984, pp. 165-215

[6] Kielkopf, Ch. F., 2uantifiers in Ontology, Studia Logica,1977, xxxvI, 4, pp. $30 \mathrm{I}-307$

[7] Küng, G., The Meaning of the 2uantifiers in the Logic of Leśniereski, Studia Logica, I977, xxxvi, 4, pp. 309-322

[8] Lejewski, C., Logic and Existence, Leśniereskis Systems. Ontology and Mereology, Editors: Jan T. J. Srzednicki, V. F. Rickey, J. Czelakowski, Polish Academy of Sciences, Institute of Philosophy and Sociology, Matrinus Nijhoff Publishers, The Hague, 1984 , pp. $45-58$

[9] Lejewski, C., On Leśnierwskis Ontology, Leśnierwski’s Systems. Ontology and Mereology, Editors: Jan T. J. Srzednicki, V. F. Rickey, J. Czelakowski, Polish Academy of Sciences, Institute of Philosophy and Sociology, Matrinus Nijhoff Publishers, The Hague, 1984, pp. 123-149

[1o] Lejewski, C., Systems of Leśnierwski's Ontology with the Functor of Weak Inclusion as the only Primitive Term, Studia Logica, 1977, Xxxv I, 4, pp. 323-349

[II] Luschei, E., The Logical Systems of Lesniereski, North-Holland Publishing Company, Amsterdam, 1962

[12] Marcus, R. B., Interpreting 2uantification, Inquiry, vol. 6 (1972), pp. 252-259

[13] Pietruszczak, A. Bezkwantyfikatorowy Rachunek Nazw. Systemy i ich Metateoria., Wydawnictwo Adam Marszałek, Toruń, I99I

[I4] Poli, R., Libardi, M., Logic, theory of science, and metaphysics according to Stanistare Leśniewski, Grazen Philosophische Studien 57, Graz, 1999, pp. 183-219

[15] Rickey, V. F., Interpretations of Lesniereskis Ontology, Dialectica 39.3 (1985), pp. I8I-I92.

[16] Simons P. M., A Semantics for Ontology, Dialectica 29.3 (1985), pp. 193-216

[17] Słupecki, J., S. Leśniereskis Calculus of Names, Leśnierwski’s Systems. Ontology and Mereology, Editors: Jan T. J. Srzednicki, V. F. Rickey, J. Czelakowski, Polish Academy of Sciences, Institute of Philosophy and Sociology, Matrinus Nijhoff Publishers, The Hague, 1984, pp. 59-122

[18] Słupecki, J., St. Leśniereski's Protothetics, [in:] Studia Logica I (1953), reprinted in J. T. J. Srzednicki, Z. Stachniak (eds.), Leśniereskis'Systems. Protothetic, Kluwer Academic Publishers, Dordrecht, 1998, pp. 84-152

[19] Sobociński, B., Lésnierwskis Analysis of Russell's Paradox, Leśnierwskis Systems. Ontology and Mereology, Editors: Jan T. J. Srzednicki, V. F. Rickey, J. Czelakowski, Polish Academy of Sciences, Institute of Philosophy and Sociology, Matrinus Nijhoff Publishers, The Hague, 1984, pp. 15-44 
[20] Takeuti, G., Zaring, W. M., Axiomatic Set Theory, Springer-Verlag, New York, I973

[21] Urbaniak, R., On Ontological Functors of Leśnieweskis Elementary Ontology, to appear in Reports on Mathematical Logic, vol. 40, 2006

[22] Whitehead, A. N., Russell B., Principia Mathematica, vol. I, Cambridge University Press, Cambridge, I9IO

[23] Woleński, J., Filozoficzna Szkoła Lwowesko-Warszareska, Pwn, Warszawa, 1985 
The Australasian fournal of Logic (ISSN 1448-5052) disseminates articles that significantly advance the study of logic, in its mathematical, philosophical or computational guises. The scope of the journal includes all areas of logic, both pure and applied to topics in philosophy, mathematics, computation, linguistics and the other sciences.

Articles appearing in the journal have been carefully and critically refereed under the responsibility of members of the Editorial Board. Only papers judged to be both significant and excellent are accepted for publication.

The journal is freely available at the journal website at

$$
\text { http://www.philosophy.unimelb.edu.au/ajl/ }
$$

All issues of the journal are archived electronically at the journal website.

SuBSCRIPTIONS Individuals may subscribe to the journal by sending an email, including a full name, an institutional affiliation and an email address to the managing editor at ajl-editors@unimelb.edu.au Subscribers will receive email abstracts of accepted papers to an address of their choice. For institutional subscription, please email the managing editor at ajl-editors@unimelb.edu.au.

Complete published papers may be downloaded at the journal's website at http: //www.philosophy.unimelb.edu.au/ajl/ The journal currently publishes in pdf format.

Submission The journal accepts submissions of papers electronically. To submit an article for publication, send the $\mathrm{LT}_{\mathrm{E}} \mathrm{X}$ source of a submission to a member of the editorial board. For a current list of the editorial board, consult the website.

The copyright of each article remains with the author or authors of that article. 